\title{
MAXIMUM PRINCIPLE FOR ELLIPTIC OPERATORS AND APPLICATIONS
}

\section{PRINCIPE DU MAXIMUM POUR OPÉRATEURS ELLIPTIQUES ET APPLICATIONS}

\author{
Rabah TAHRAOUI ${ }^{\mathrm{a}, \mathrm{b}}$ \\ ${ }^{a}$ CEREMADE, Université Paris 9, Dauphine, UMR 7534, place du Maréchal de Lattre de Tassigny, \\ 75775 Paris cedex 16, France \\ ${ }^{\mathrm{b}}$ I.U.F.M. Rouen, 2, rue du Tronquet, 76131 Mont-St-Aignan, France
}

Received 13 July 2000, revised 17 July 2001

Abstract. - Let $f$ be a signed function defined on some bounded domain $\Omega$. We give sufficient conditions ensuring the positivity of $u$, solution of the following equation:

$$
-\Delta u=f \quad \text { in } \Omega,\left.\quad u\right|_{\partial \Omega}=0 .
$$

(C) 2002 L'Association Publications de l'Institut Henri Poincaré. Published by Elsevier B.V. All rights reserved AMS classification: $35 \mathrm{~J} 25 ; 35 \mathrm{~K} 20 ; 35 \mathrm{~B} 50$

RÉSUMÉ. - Soit une fonction $f$ de signe non constant sur un domaine borné $\Omega$. On donne des conditions suffisantes assurant la stricte positivité de la solution de l'équation :

$$
-\Delta u=f \quad \text { dans } \Omega,\left.\quad u\right|_{\partial \Omega}=0 .
$$

(C) 2002 L'Association Publications de l'Institut Henri Poincaré. Published by Elsevier B.V. All rights reserved

\section{Introduction}

We are concerned with maximum principle for linear second order partial differential equations. The maximum principle is a mathematical tool which is used frequently in many fields of mathematics. For instance, its use is often essential in the study of nonlinear partial differential equations: existence and qualitative result [9,5]. In this field, the literature is well documented and our reference list is by no means exhaustive. For instance, we refer to $[5,11,10]$, for precise informations. 
For sake of clarity, let us recall the following definition: Let $\Omega$ be an open bounded subset of $\mathbb{R}^{N}$; we say that the operator

$$
A=-\sum_{i, j} a_{i j} \frac{\partial^{2}}{\partial x_{i} \partial x_{j}}+\sum_{i} b_{i} \frac{\partial}{\partial x_{i}}+c
$$

has the maximum principle property if, for any nonnegative function $f$, the solution $u$ of - (if it exists)

$$
\left\{\begin{array}{l}
A u=f \quad \text { in } \Omega \\
\left.u\right|_{\partial \Omega}=0
\end{array}\right.
$$

is positive.

In the present paper, we deal with the following natural question: let $f$ be a function defined on $\Omega$ such that

$$
\begin{aligned}
& f^{+}(x)=\sup (f(x), 0) \not \equiv 0, \\
& f^{-}(x)=\sup (-f(x), 0) \not \equiv 0 .
\end{aligned}
$$

Is the solution $u$ of $(*)$ positive? We are looking for assumptions on $f$ which imply that the answer to this question is positive. In a simple but significant setting the main result of the present paper can be stated as follows: Let $\Omega$ be a ball $B(0, R)$ of $\mathbb{R}^{N}, f$ a function defined on $\Omega$ such that

$$
f(x) \geqslant g(|x|)=g^{+}(|x|)-g^{-}(|x|)
$$

where $g$ is a radial function. Assume that

$$
\int_{0}^{R} \frac{d \sigma}{\sigma^{N-1}} \int_{0}^{\sigma^{N} / N}\left[\left(g^{+}\right)_{*}-\left(g^{-}\right)^{*}\right](s) d s>0,
$$

where, for any function $h, h_{*}$ (respectively $h^{*}$ ) stands for the nondecreasing (respectively the nonincreasing) rearrangement of $h$, with respect to the measure $\mu$ defined by

$$
\mu(E)=\int_{E} s^{N-1} d s
$$

for any measurable subset $E$ of $[0, R]$.

THEOREM 1. - Under hypothesis (a) and (b), the solution $u$ of

$$
\left\{\begin{array}{l}
-\Delta u=f \quad \text { in } B(0, R), \\
\left.u\right|_{\partial \Omega}=0,
\end{array}\right.
$$

is positive.

To be more clear and explicit, let us give the meaning of our result throughout the following particular case [14]. Suppose that $\Omega \subset \mathbb{R}^{N}$. Let $B\left(0, r_{0}\right)$ and $B(0, R)$ be two 
open balls such that

$$
B\left(0, r_{0}\right) \subset B(0, R) \subseteq \Omega .
$$

Assume that

$$
f(x) \in\{-\alpha, \beta\} \quad \text { and } \quad\{x \in \Omega \mid f(x)=-\alpha\} \subseteq B\left(0, r_{0}\right),
$$

where $\alpha$ and $\beta$ are two positive real numbers. Then the solution $u$ of

$$
\left\{\begin{array}{l}
-\Delta u=f \quad \text { in } \Omega \\
\left.u\right|_{\partial \Omega}=0,
\end{array}\right.
$$

is positive if we have the following inequalities

$$
\begin{array}{ll}
1+\frac{\alpha}{\beta} \leqslant\left(\frac{R}{r_{0}}\right)^{N} \frac{N-2}{N\left(\frac{R}{r_{0}}\right)^{N-2}-2} \quad \text { if } N>2, \\
1+\frac{\alpha}{\beta} \leqslant\left(\frac{R}{r_{0}}\right)^{2} \frac{1}{1+2 \log \left(\frac{R}{r_{0}}\right)} \quad \text { if } N=2 .
\end{array}
$$

In this work the main difficulty occurs if the zero order coefficient $c$ of operator $A$ is not equal to zero; it is enough to state our results in the model case

$$
\left\{\begin{array}{l}
-\Delta u+c . u=f \quad \text { in } \Omega, \\
\left.u\right|_{\partial \Omega}=0 .
\end{array}\right.
$$

In fact, up to some technical computations, we can obtain our results for general operator $A$. We will apply our maximum principle result to many frameworks:

(i) We will state that the solution of

$$
\left\{\begin{array}{l}
-\Delta u-c|u|=f \quad \text { in } B(0, R) \\
\left.u\right|_{\partial B}=0
\end{array}\right.
$$

has a positive radial average if the radial average of $f$ verifies some natural hypothesis.

(ii) We will study the positivity of the solution of the following semi-linear problem, without monotonicity or positivity hypothesis on $f$ :

$$
\left\{\begin{array}{l}
-\Delta u=f(x, u) \quad \text { in } \Omega \\
\left.u\right|_{\partial \Omega}=0 .
\end{array}\right.
$$

(iii) We will state the positivity of the solution of the fourth order problem

$$
\left\{\begin{array}{l}
-\Delta^{2} u=f \quad \text { in } \Omega \\
\left.u\right|_{\partial \Omega}=\left.\Delta u\right|_{\partial \Omega}=0
\end{array}\right.
$$

For a first result about the positivity of the solution of this equation we can see [14] where $\Omega$ is a ring.

(iv) By a negative result we will prove that the main hypothesis used in this paper is not related to the method. 
(v) Our method works in the case of parabolic equation.

(vi) Localization of critical point [16]: here we will explain how to use our main result to study the star-shapedness property of the level sets of solutions to various p.d.e. in the case $\Omega$ simply connected. Such results are already known in the case $\Omega=\Omega_{1} \backslash \Omega_{2}$, $\bar{\Omega}_{2} \subset \Omega_{1}$, where $\Omega_{i}$ is, for instance, convex: [7,4,3]. And let us point out that the results of the papers mentionned above do not apply in the case $\Omega$ simply connected.

\section{Setting of the problem, notations and hypothesis}

Let $\Omega$ be an open bounded and regular subset of $\mathbb{R}^{N}$. We suppose that $\Omega$ is connected. Let us consider the solution $u$ of the following equation

$$
\left\{\begin{array}{l}
-\Delta u+c . u=f \quad \text { in } \Omega \\
\left.u\right|_{\partial \Omega}=0 .
\end{array}\right.
$$

We assume that the operator $-\Delta+c . I$ satisfies the maximum principle on $\Omega$ (for more details about maximum principle cf. $[9,10])$. Let us denote by $E_{f}$ the negative support of $f$, i.e., the set

$$
E_{f}=\{x \in \Omega \mid f(x)<0\} .
$$

We assume that

$$
\bar{E}_{f} \subset \Omega \text {. }
$$

Let $\varphi$ and $h$ be two functions defined on $\Omega$ and such that $0 \leqslant \varphi(x) \leqslant T$. For any $x$ in $\Omega$, set

$$
\begin{aligned}
g(x)=\sup \{k(\varphi(x)) \mid & k(.) \text { continuous function } \\
& \text { defined on }[0, T] \text { s.t. } k o \varphi(.) \leqslant h(.) \text { on } \Omega\} .
\end{aligned}
$$

There exists a function $h_{\varphi}($.$) defined on [0, T]$ s.t.

$$
h_{\varphi}(\varphi(x))=g(x) \leqslant h(x) \quad \forall x \in \Omega .
$$

In the same way, we define, for any $x$ in $\Omega$,

$$
\begin{aligned}
k(x)=\inf \{l(\varphi(x)) \mid & l(.) \text { continuous function } \\
& \text { defined on }[0, T] \text { s.t. } \operatorname{lo} \varphi(.) \geqslant h(.) \text { on } \Omega\} .
\end{aligned}
$$

We define, on $[0, T]$, a function $h^{\varphi}($.$) such that$

$$
h^{\varphi}(\varphi(x))=k(x) \geqslant h(x) \quad \forall x \in \Omega .
$$

\section{DEFINITION 1.-}

1. Function $h_{\varphi}($.$) defined by (3) and (4.1) is called estimate from below of h$ with respect to $\varphi$, on $\Omega$. 
2. Function $h^{\varphi}$ defined by (4.2) is called estimate from above of $h$ with respect to $\varphi$, on $\Omega$.

3. Function $\varphi$ plays an important role in this study. We call it shape function.

Remarks 1.-

(i) Let $f$ be positive function. Then $\int_{\Omega} f_{\varphi} d x<+\infty$ if $\int_{\Omega} f d x<+\infty$.

(ii) $\int_{\Omega}(|f|)^{\varphi} d x<+\infty$ entails that $\int_{\Omega}\left|f_{\varphi}\right| d x<+\infty$.

(iii) $f_{\varphi}$ and $f^{\varphi}$ are bounded if $f$ is bounded.

(iv) The hypothesis $\int_{\Omega}\left|f_{\varphi}\right| d x<+\infty$ assumed in (6) Section 3, is necessary to construct positive subsolution of (1) which is the main idea of our paper; this is showed by the following example:

$$
\begin{aligned}
& \Omega=B(0, R) \subseteq \mathbb{R}^{2} \varphi(x)=|x|, \quad x=\left(x_{1}, x_{2}\right), \\
& f(x)= \begin{cases}-1 /\left|x_{1}\right|^{\alpha}, & \text { if }\left|x_{1}\right| \leqslant r_{0}, x \in B(0, R), \\
1 /\left|x_{1}\right|^{\alpha}, & \text { if }\left|x_{1}\right|>r_{0}, x \in B(0, R),\end{cases}
\end{aligned}
$$

with $0<\alpha<\frac{1}{2}$. Function $f$ belongs to $L^{2}(\Omega)$ and $f_{\varphi}(|x|) \equiv-\infty$. In this case, our method does not work.

DEFINITION 2. - Let $r$ and s be two bounded and positive functions defined on $[0, T]$. Let us set

$$
\lambda(r, s, 0, T)=\inf \left[\int_{0}^{T} r \psi^{\prime 2} d x .\left(\int_{0}^{T} s \psi^{2} d x\right)^{-1} \mid \psi \in H^{1}(] 0, T[), \psi(T)=0\right] .
$$

The goal of this study is to give some sufficient conditions on $f$ to ensure the positivity of the solution $u$ of Eq. (1). These conditions are nearly necessary. Roughly speaking, our fundamental idea is to construct a positive subsolution of (1) which is of the form $v \circ \varphi($.$) , where v$ is defined on $[0, T]$. For the sake of clarity, we present, in a first step, our idea in a simple framework: $\varphi(x)=|x|$ is a radial function and the operator in (1) is such that $c \equiv 0$. We will see that the case $c \not \equiv 0$ is more complex.

\section{The shape function is radial}

To simplify the technical details of our work, we assume that

$$
\begin{aligned}
& \varphi(x)=|x| \quad \forall x \in \mathbb{R}^{N}, \\
& c(x)=0 \quad \forall x \in \Omega .
\end{aligned}
$$

And roughly speaking we look for positive subsolution $\mathcal{W}$ of (1) radial in $B(O, R)$ :

$$
\left\{\begin{array}{l}
\mathcal{W}(x):=w(\varphi(x))=w(|x|) \quad \text { in } B(O, R) \subseteq \Omega \\
-\Delta \mathcal{W} \leqslant f \quad \text { in } H^{-1}(\Omega) \\
\left.\mathcal{W}\right|_{\partial \Omega}=0, \quad \mathcal{W} \geqslant 0 \quad \forall x \in \Omega
\end{array}\right.
$$

where $R$ is the radius of the largest ball $B=B(O, R)$ enclosed in $\bar{\Omega}$. For simplicity, we assume that $O$ is the center of the ball $B$. We denote

$$
f_{R}(x)=f(x) \quad \forall x \in B(O, R),
$$


and we assume the following hypothesis

$$
\begin{gathered}
\int_{B}\left|\left(\widetilde{f}_{R}\right)_{\varphi}\right| d x<+\infty, \\
\left\{\begin{array}{l}
\exists r_{0}, 0<r_{0}<R \quad \text { s.t. } \quad E_{f} \subseteq B\left(0, r_{0}\right), \quad \text { and } \\
G(R, f)=\int_{0}^{R} \frac{1}{r^{N-1}} \int_{0}^{r} s^{N-1}\left(\widetilde{f}_{R}\right)_{\varphi}(s) d s d r>0
\end{array}\right.
\end{gathered}
$$

where $E_{f}=\{x \in \Omega \mid f(x)<0\}$ and where $\left(\widetilde{f}_{R}\right)_{\varphi}$ is given by Definition 1, and

$$
\begin{aligned}
& \widetilde{f}_{R}(x)=f(x) \quad \forall x \in B(O, R) \backslash\left[B\left(O, r_{0}\right) \backslash E_{f}\right], \\
& \widetilde{f}_{R}(x)=0 \quad \text { if } x \in B\left(O, r_{0}\right) \backslash E_{f} .
\end{aligned}
$$

$E_{f} \subset B\left(0, r_{0}\right)$ is called the localization hypothesis of negative support $E_{f}$.

Remarks 2.-

(1) Let us point out that hypothesis (6) is satisfied for any $f$ bounded from below.

(2) We can relax (7) by $G(R, f)=0$. In this case, the result of Theorem 1 could be some what weaker. This situation will be studied in Theorem 2 for ellipsoïdal coordinates.

LEMMA 1. - Let us consider the function

$$
w(r)=\int_{r}^{R} \frac{1}{\sigma^{N-1}}\left[\int_{0}^{\sigma} s^{N-1}\left(\tilde{f}_{R}\right)_{\varphi}(s) d s\right] d \sigma .
$$

$w$ is positive and satisfies Eq. (8) written here after.

Proof. - From (7) we have:

$$
\begin{array}{ll}
\left(\widetilde{f}_{R}\right)_{\varphi}(r) \leqslant 0, & 0 \leqslant r \leqslant r_{0}, \\
\left(\widetilde{f}_{R}\right)_{\varphi}(r) \geqslant 0, & r_{0}<r \leqslant R .
\end{array}
$$

Then there exists some $\bar{r}>r_{0}$ such that $w^{\prime}(\bar{r})=0, w^{\prime}(r) \geqslant 0 \forall r, 0 \leqslant r \leqslant \bar{r}, w^{\prime}(r) \leqslant 0$, $\forall r, \bar{r} \leqslant r \leqslant R$. Since $w(0) \geqslant 0$ from (7) and $w(R)=0$, we obtain that

$$
w(r) \geqslant 0 \quad \forall r, 0 \leqslant r \leqslant R .
$$

To achieve the proof, it is easy to verify that $w$ is solution of (8) here after.

Indeed let us consider $w$ the solution of

$$
\left\{\begin{array}{l}
-\frac{1}{r^{N-1}} \frac{d}{d r}\left(r^{N-1} \frac{d w}{d r}\right)=g(r)=\left(\widetilde{f}_{R}\right)_{\varphi} \quad \text { in } B(0, R), \\
\frac{d w}{d r}(0)=0, \quad w(R)=0 .
\end{array}\right.
$$


It is easy to see that

$$
w(r)=\int_{r}^{R} \frac{1}{\sigma^{N-1}} \int_{0}^{\sigma} s^{N-1} g(s) d s d \sigma .
$$

From hypothesis (7), we have $w(r)>0$ for any $r, 0<r<R$. Let us denote

$$
\mathcal{W}(x)=w(|x|) \quad \forall x \in B(O, R)
$$

We have

$$
\left\{\begin{array}{l}
-\Delta \mathcal{W}=g \leqslant f \quad \text { in } B(O, R), \\
\left.\mathcal{W}\right|_{\partial B}=0
\end{array}\right.
$$

We are going to extend inequality (9) to $\Omega$, in some sense. Let $\tilde{g}$ be a function defined as follows

$$
\tilde{g}(x)= \begin{cases}g(|x|) & \forall x \in B(O, R), \\ 0 & \forall x \in \Omega \backslash B(O, R),\end{cases}
$$

and let us denote

$$
\widetilde{\mathcal{W}}(x)= \begin{cases}\mathcal{W}(x) & \text { if } x \in \overline{B(O, R)} \\ 0 & \text { if } x \in \Omega \backslash \overline{B(O, R)}\end{cases}
$$

$\widetilde{\mathcal{W}}$ belongs to $H_{0}^{1}(\Omega)$. From extension lemma (cf. Appendix C) we obtain:

$$
\left\{\begin{array}{l}
-\Delta \widetilde{\mathcal{W}} \leqslant \widetilde{g} \\
\widetilde{\mathcal{W}} \in H_{0}^{1}(\Omega)
\end{array} \quad \text { in } H^{-1}(\Omega)\right.
$$

and

$$
\left\{\begin{array}{l}
-\Delta(\widetilde{\mathcal{W}}-u) \leqslant \widetilde{g}-f \quad \text { in } H^{-1}(\Omega), \\
\widetilde{\mathcal{W}}-u \in H_{0}^{1}(\Omega) .
\end{array}\right.
$$

As $\widetilde{g}(x)-f(x) \leqslant 0 \forall x \in \Omega, \widetilde{g}()-.f(.) \not \equiv 0$, we obtain, from Stampacchia result's [11], that

$$
\widetilde{\mathcal{W}}(x)-u(x)<0 \quad \forall x \in \Omega,
$$

i.e., $u(x)>0 \forall x \in \Omega$, since from hypothesis (7) we have

$$
\widetilde{\mathcal{W}}(x) \geqslant 0 \quad \forall x \in \Omega \text {. }
$$

So we have proved the following result.

THEOREM 1. - Under hypothesis (6) and (7), the solution $u$ of (1) is positive in $\Omega$.

A simple and illustrative example (cf. $[13,14])$. - In order to give a precise idea of our result, we propose a very simple but significant example. Let $u$ be the solution of

$$
\left\{\begin{array}{l}
-\Delta u=f \quad \text { in } \Omega=B(O, R) \subset \mathbb{R}^{N}, \\
\left.u\right|_{\partial \Omega}=0,
\end{array}\right.
$$


where $f(x) \in\{\alpha, \beta\}$ a.e. $x \in \Omega, \alpha$ and $\beta$ being two real numbers, $\alpha<0, \beta>0$. We assume that

$$
E_{f}=\{x \in \Omega \mid f(x)=\alpha\} \subseteq B\left(O, r_{0}\right)
$$

where $\alpha, \beta, r_{0}$ and $R$ are such that:

$$
\begin{cases}1-\frac{\alpha}{\beta} \leqslant\left(\frac{R}{r_{0}}\right)^{N} \frac{N-2}{N\left(\frac{R}{r_{0}}\right)^{N-2}-2} & \text { if } N>2, \\ 1-\frac{\alpha}{\beta} \leqslant\left(\frac{R}{r_{0}}\right)^{2} \cdot \frac{1}{1+2 \log \left(\frac{R}{r_{0}}\right)} \quad \text { if } N=2 .\end{cases}
$$

Theorem 1 states that $u$ is positive. Relations (10.1) mean that if the set $E_{f}$ is "sufficently far" from the boundary of $\Omega$ and if $|\alpha|$ is not "too large" with respect to $\beta$, then $u$ is positive. If $E_{f}=B\left(O, r_{0}\right),(10.1)$ is necessary to obtain $u$ positive.

\section{The shape function is ellipsoïdal}

In Section 3, we used radial coordinates. These coordinates are not always well adapted to the geometry of $\Omega$. So we use in the following ellipsoïdal coordinates as in [17]. For this let us give a familly of parametrized ellipsoïds $B(\rho)$ centred at $O$. We denote the boundary of such an ellipsoïd by $\partial B(\rho)$. This boundary $\partial B(\rho)$ is characterised by the following equation:

$$
\forall x \in \partial B(\rho), \quad \sum_{j} x_{j}^{2} /\left(\rho+q_{j}\right)=1, \quad q_{j} \geqslant 0, \forall j=1,2, \ldots, N,
$$

where one of the $q_{j}$ is zero and $\rho$ is a positive parameter. In the sequel, we set

$$
m_{j}=m_{j}(\rho)=\left(\rho+q_{j}\right)^{1 / 2}, \quad \forall j=1,2, \ldots, N .
$$

Let us notice that we have

$$
\rho=\inf _{j}\left(m_{j}^{2}\right) .
$$

If the ellipsoï $B(\rho)$ is not degenerate, i.e., when $\rho>0, \rho$ is given by an implicit function $\varphi($.) of $x: \rho=\varphi(x)$. The level sets of $\varphi($.$) represent boundaries of B(\rho)$, i.e.,

$$
\partial B(\rho)=\{x \mid \varphi(x)=\rho\} \quad \text { for some level } \rho .
$$

From the computations of [17] we obtain the following relations:

$$
\Delta \varphi(x)=2 \widehat{\beta}(x) \psi(x, \rho), \quad \rho=\varphi(x), \quad|\nabla \varphi(x)|^{2}=2 \psi(x, \rho),
$$




$$
\left\{\begin{array}{l}
\widehat{\beta}(x)=\frac{1}{2} \sum_{j} \frac{1}{\varphi(x)+q_{j}}=\theta(\varphi(x)), \\
\widehat{\beta}(x)=\frac{\Delta \varphi(x)}{|\nabla \varphi(x)|^{2}} \\
\theta(t)=\frac{1}{2} \sum_{j} \frac{1}{t+q_{j}}, \\
\psi(x, \rho)=2\left[\sum_{j} x_{j}^{2} /\left(\rho+q_{j}\right)^{2}\right]^{-1}
\end{array}\right.
$$

For more details, cf. [17]. Up to a translation, we can, to simplify, assume that the origin $O$ belongs to $\Omega$ and is the center of the ellipsoïds. Let us set

$$
\begin{gathered}
\tilde{\gamma}(x)=\frac{c(x)}{|\nabla \varphi(x)|}, \quad \gamma(x)=\frac{c^{+}(x)}{|\nabla \varphi(x)|} \quad \forall x \in \Omega \text { s.t. }|\nabla \varphi(x)| \neq 0 \\
T=\sup \left\{\rho \in \mathbb{R}^{+} \mid B(\rho) \subseteq \Omega\right\} .
\end{gathered}
$$

Let us remark that $B(\rho)$ is an increasing family of subsets of $\Omega$ and $T=\left.\varphi\right|_{\partial B(T)}=$ $\sup \{\varphi(x) \mid x \in B(T)\}$. We suppose that

$$
|\tilde{\gamma}(x)|=\frac{|c(x)|}{|\nabla \varphi(x)|} \leqslant M \quad \forall x \in \Omega,
$$

and

$$
\left.f|| \nabla \varphi\right|^{2} \text { belongs to } L^{1}(\Omega) \text {. }
$$

Remarks about hypothesis (15). - The following example shows that (15) is somewhat necessary.

Consider $\left.b(x)=1 /\left(x-x_{0}\right)^{2} \forall x \neq x_{0}, x \in\right] 0,1\left[\right.$, where $\left.x_{0} \in\right] 0,1\left[\right.$ is fixed. Let $u_{\varepsilon}$ be the solution of

$$
\left\{\begin{array}{l}
\left.-u_{\varepsilon}^{\prime \prime}+b_{\varepsilon} \cdot u_{\varepsilon}=g, \quad g \geqslant 0 \text { on }\right] 0,1[ \\
u_{\varepsilon}(0)=u_{\varepsilon}(1)=0, \quad g \text { bounded }
\end{array}\right.
$$

with

$$
b_{\varepsilon}(x)= \begin{cases}1 / \varepsilon^{2} & \text { if } x \in\left[x_{0}-\varepsilon, x_{0}+\varepsilon\right]=I_{\varepsilon}, \\ b(x) & \text { if } x \in] 0,1\left[-I_{\varepsilon} .\right.\end{cases}
$$

Then $u_{\varepsilon}\left(x_{0}\right) \rightarrow 0$ as $\varepsilon \rightarrow 0$. Indeed, arguing by contradiction, i.e., suppose that there exists $\alpha>0$ such that for any $\varepsilon, 0<\varepsilon<\varepsilon_{0}$

$$
\inf \left\{u_{\varepsilon}(x) \mid x \in\left[x_{0}-\varepsilon_{0}, x_{0}+\varepsilon_{0}\right]\right\} \geqslant \alpha
$$


We have:

$$
\int_{0}^{1}\left|u_{\varepsilon}^{\prime}\right|^{2} d x+\int_{0}^{1} b_{\varepsilon}\left(u_{\varepsilon}\right)^{2} d x \leqslant\|g\|_{\infty} \cdot\left\|u_{\varepsilon}\right\|_{L^{2}},
$$

from which we deduce

$$
\int_{0}^{1} b_{\varepsilon}\left(u_{\varepsilon}\right)^{2} d x \leqslant c .
$$

Thus

$$
0<\frac{\alpha^{2}}{\varepsilon^{2}} \int_{x_{0}-\varepsilon}^{x_{0}+\varepsilon} d x \leqslant \int_{x_{0}-\varepsilon}^{x_{0}+\varepsilon} b_{\varepsilon}\left(u_{\varepsilon}\right)^{2} \leqslant c \quad \forall \varepsilon>0
$$

or again

$$
2 \alpha^{2} \leqslant \varepsilon . c
$$

which is not possible for $\varepsilon$ small enough. Recall that $\gamma(x)=c^{+}(x) /|\nabla \varphi(x)|$ allows us to define $\gamma^{\varphi}$ which helps to obtain positive solution $a($.$) of$

$$
\left\{\begin{array}{l}
-\frac{1}{\beta}\left(\beta a^{\prime}\right)^{\prime}+\gamma^{\varphi} a=0 \quad \text { on }[0, T], \\
a^{\prime}(0)=0, \quad a(T)=1,
\end{array}\right.
$$

where

$$
\beta^{2}(s)=\prod_{j=1}^{N}\left(s+q_{j}\right)
$$

and $q_{j} \geqslant 0$ are some constants. From example (17), we see easily that if $\gamma^{\varphi}$ is not bounded, the solution $a($.$) of the above equation can vanish in [0, T]$. We will also see later in Proposition 1 , that $a()>$.0 on $[0, T]$ is essential to construct a positive subsolution of (1). So taking into account the above example, hypothesis (15) is quite natural; indeed if $c() \geqslant 0,.(15)$ is some what necessary to our construction because our method requires to have $a()>$.0 on $[0, T]$. And if $E_{c}=\{x \in \Omega \mid c(x)<0\}$ is such that $\left|E_{c}\right|>0, \overline{E_{c}} \subset \Omega$, we choose suitably the origin $O$ in $E_{c}$. Thus $\gamma(x)$ is bounded if $c(x)$ is bounded, since $O$ is the center of $B(\rho)$. In a first step we study positivity of the solution $v$ of

$$
\left\{\begin{array}{l}
-\Delta v+c^{+} . v=f \quad \text { in } \Omega \\
\left.v\right|_{\partial \Omega}=0
\end{array}\right.
$$

In a second step, it sufficies to remark that, if $v$ is nonnegative, we have

$$
u \geqslant v \quad \text { in } \Omega
$$

which gives the expected result. Finally, let us point out that if $v$ is nonpositive we also have $u \leqslant v$ in $\Omega$. These remarks show that, instead of (1), the significant problem that we have to consider is problem (16.1). So we will study it subsequently. 
Remark. - Let us point out that if

$$
|\{x \in \Omega \mid c(x)<0\}|>0
$$

it is necessary to suppose

$$
\left\|c^{-}\right\|_{L^{\infty}(\Omega)}<\lambda_{1}(\Omega, \Delta)
$$

to have existence and classical maximum principle for $(1) . \lambda_{1}(\Omega, \Delta)$ is the first eigenvalue of $\Delta$, in $\Omega$, with Dirichlet boundary condition.

Remarks about hypothesis (16). - First of all, let us recall that $O$ is the center of the ellipsoïds $B(\rho)$. If $f /|\nabla \varphi|^{2}$ does not belong to $L^{1}(\Omega)$, we can proceed by approximation procedure. That is to say, since from (2) $\bar{E}_{f} \subset \Omega$, we choose the origin $O$ in $\Omega \backslash \bar{E}_{f}$ and study the positivity of the solution $v_{\varepsilon}$ of

$$
\left\{\begin{array}{l}
-\Delta v_{\varepsilon}+c^{+} . v_{\varepsilon}=f_{\varepsilon} \quad \text { in } \Omega \\
\left.v_{\varepsilon}\right|_{\partial \Omega}=0
\end{array}\right.
$$

where

$$
f_{\varepsilon}(x)= \begin{cases}0 & \text { if } x \in B(0, \varepsilon), \\ f(x) & \text { if } x \in \Omega \backslash \overline{B(0, \varepsilon)},\end{cases}
$$

for $\varepsilon>0$ small enough. Next we pass to the limit as $\varepsilon$ goes to zero and the expected result follows for (16.1), if the necessary hypothesis are satisfied.

The following lemma is necessary to understand the hypothesis that we will assume and which establishes the quantitative link between functions $f^{+}=\sup (f, 0), f^{-}=$ $-\inf (f, 0)$ and their respective supports.

Lemma 2. - There exists a unique $\tau, 0<\tau$, such that

$$
\begin{aligned}
& \left.\frac{1}{t^{2}} \cdot\left(\frac{\pi}{2}\right)^{2}=\lambda(1,1,0, t)>\left(\left\|\gamma^{\varphi}\right\|_{\infty}\right)^{2} \cdot \frac{t^{2}}{2} \quad \forall t \in\right] 0, \tau[, \\
& \lambda(1,1,0, \tau)=\left(\left\|\gamma^{\varphi}\right\|_{\infty}\right)^{2} \cdot \frac{\tau^{2}}{2} .
\end{aligned}
$$

Proof. - The function $t \rightarrow \lambda(1,1,0, t)$ is decreasing and $\lambda(1,1,0, t) \rightarrow+\infty$ as $t$ goes to $0^{+}$. Therefore, there exists $\tau>0$ such that

$$
\lambda(1,1,0, \tau)=\left(\left\|\gamma^{\varphi}\right\|_{\infty}\right)^{2} \cdot \frac{\tau^{2}}{2}
$$

and

$$
\left.\lambda(1,1,0, t)>\left(\left\|\gamma^{\varphi}\right\|_{\infty}\right)^{2} \cdot \frac{t^{2}}{2} \quad \forall t \in\right] 0, \tau[.
$$

The equality $\lambda(1,1,0, t)=\left(\frac{\pi}{2}\right)^{2} \frac{1}{t^{2}}$ follows from a classical computation.

In some sense, Lemma 3 will show that the use of ellipsoïdal coordinates is somewhat "miraculous" since ellipsoïdal computations are nearly similar to radial ones. 
LEMMA 3. - We have

$$
\widehat{\beta}_{\varphi}(t)=\widehat{\beta}^{\varphi}(t)=\theta(t)=\frac{\alpha}{t}+\delta(t), \quad \forall t \in[0, T],
$$

where $\alpha$ is a real constant, $\geqslant \frac{1}{2}$ and $\delta($.$) is a positive and bounded function defined on$ $[0, T]$.

Proof. - Let $i_{1}, i_{2}, \ldots, i_{k}, k \geqslant 1$, be indexes belonging to $\{1,2, \ldots, N\}$ such that

$$
\rho=\inf _{j} m_{j}^{2}=m_{i_{1}}^{2}=m_{i_{2}}^{2}=\cdots=m_{i_{k}}^{2} .
$$

Therefore

$$
q_{i_{1}}=q_{i_{2}}=\cdots=q_{i_{k}}=0, \quad q_{j}>0 \quad \forall j \neq i_{1}, \ldots, i_{k} .
$$

So

$$
\theta(t)=\frac{1}{2} \frac{k}{t}+\frac{1}{2} \sum_{j \notin\left\{i_{1}, \ldots, i_{k}\right\}} \frac{1}{t+q_{j}}
$$

i.e.,

$$
\theta(t)=\frac{k}{2} \cdot \frac{1}{t}+\delta(t)
$$

In the sequel we need to use the following ordinary differential equation and its solution $a()=.a_{t}($.$) :$

$$
\left\{\begin{array}{l}
\left.-\frac{1}{\beta(s)}\left(\beta(s) a^{\prime}(s)\right)^{\prime}+\gamma^{\varphi}(s) a(s)=0 \quad \text { in }\right] 0, t[ \\
a^{\prime}(0)=0, \quad a(t)=1,
\end{array}\right.
$$

where

$$
\beta^{2}(s)=\prod_{j=1}^{N}\left(s+q_{j}\right)
$$

If there is no ambiguity, we denote the solution $a_{t}$ (.) of (20) by $a($.$) . In Appendix \mathrm{B}$, we prove that (20) has a unique solution $a_{t}$ (.) belonging to $C^{0}([0, t])$ such that $a(s)>0$, $\forall s 0 \leqslant s \leqslant t$. Let us recall that the positivity of $a($.$) requires (15) (cf. remarks about$ (15)). In Appendix B the reader can find some regularity results about (20). Now let us assume the following hypothesis:

(H0) Localization hypothesis of the negative support $E_{f}$. There exists $t_{0}$ such that $0<t_{0}<T$ and $E_{f} \subseteq B\left(t_{0}\right) \subset \Omega$.

(H1) There exists $\bar{t}, 0<t_{0}<\bar{t} \leqslant T$ such that

$$
\Sigma(\bar{t})=\int_{0}^{\bar{t}}\left[\int_{0}^{\sigma} a(s) \widetilde{f}_{\nabla, \varphi}(s) \beta(s) d s\right] \frac{d \sigma}{a^{2}(\sigma) \beta(\sigma)} \geqslant 0
$$


where

$$
\begin{gathered}
\tilde{f}_{\nabla, \varphi}=\left(\frac{\tilde{f}}{|\nabla \varphi|^{2}}\right)_{\varphi} \text { (cf. Definition 1), } \\
\widetilde{f}(x)=f(x) \quad \text { if } x \notin B\left(t_{0}\right) \backslash E_{f}, \quad \widetilde{f}(x)=0 \quad \text { if } x \in B\left(t_{0}\right) \backslash E_{f},
\end{gathered}
$$

$\beta$ is given by (21) and $a($.) is the unique solution of (20) in $] 0, T[$.

(H2) The operator $-\Delta+c^{+}(x) . I$ and the function shape $\varphi$ verify

$$
\left(\frac{\pi}{2}\right)^{2} \frac{1}{\bar{t}^{2}}=\lambda(1,1,0, \bar{t})>\left(\left\|\gamma^{\varphi}\right\|_{\infty}\right)^{2} \cdot \frac{\bar{t}^{2}}{2} .
$$

Remark 3 (Comments about hypothesis $\mathrm{H} 1$ and $\mathrm{H} 2)$. - If (H1) is satisfied for $\bar{t}>\tau$, given by Lemma 2, then (H1) and (H2) are not satisfied simultaneously. This means that $f^{+}$is not sufficiently preponderant with respect to $f^{-}$in a neighbourhood of $E_{f}$. And the method does not work.

THEOREM 2. - We assume (15), (16), (H0), (H1) and (H2). Then the solution $u$ of (1) satisfies the following:

(i) $u(x)>0$ if $\Sigma(\bar{t})>0$,

(ii) $u(x)>0$ if $\Sigma(\bar{t})=0, B(\bar{t}) \subset \Omega$ and $f \neq 0$ on $\Omega \backslash B(\bar{t})$,

(iii) $u(x) \geqslant 0$ if $\Sigma(\bar{t})=0$ and $B(\bar{t})=\Omega$ or $\Sigma(\bar{t})=0$ and $B(\bar{t}) \subset \Omega$ with $f \equiv 0$ on $\Omega \backslash B(\bar{t})$.

Proof of Theorem 2. - For the proof of this result, we proceed in several steps. The main idea is to construct a positive subsolution of (1) which has the same level sets as $\varphi$.

Step 1. (A differential equation, its link with (1) and its representation formula.) Let us consider the following equation

$$
\left\{\begin{array}{l}
\left.-\ell^{\prime \prime}(t)-\theta(t) \ell^{\prime}(t)+\gamma^{\varphi} \cdot \ell(t)=\widetilde{f}_{\nabla, \varphi}(t)=g(t), \quad \forall t \in\right] 0, \bar{t}[, \\
\ell^{\prime}(0)=\ell(\bar{t})=0, \quad \bar{t} \text { is given by }(\mathrm{H} 1),
\end{array}\right.
$$

where $\widetilde{f}_{\nabla, \varphi}$ is defined by (21.1), $\theta($.$) is given by Lemma 2$ and (13.1). $\gamma$ is defined by (14).

Proposition 1. - Suppose that $\gamma^{\varphi}$ belongs to $L^{\infty}(] 0, \bar{t}[)$. Then there exists a solution of (22), denoted by $\ell($.$) , and which has the following representation formula:$

$$
\ell(t)=a(t) \int_{t}^{\bar{t}} \frac{d \sigma}{a^{2} \beta(\sigma)} \int_{0}^{\sigma} a \beta \tilde{f}_{\nabla, \varphi}(s) d s
$$

with $\beta(t)=\left[\prod_{j=1}^{N}\left(t+q_{j}\right)\right]^{1 / 2}$, and where a(.) is the solution of $E q$. (20) on $] 0, T[; \bar{t}$ is given by hypothesis (H1).

Proof of Proposition 1. - From Appendix B, Eq. (20) has a continuous positive solution, denoted $a($.$) . Therefore, we can set$

$$
\ell(t)=a(t) \cdot v(t) \quad \forall t \in[0, \bar{t}]
$$


where $v($.$) is the solution of the following equation:$

$$
\left\{\begin{array}{l}
\left.-\frac{1}{\beta a}\left(a^{2} \beta v^{\prime}\right)^{\prime}=\widetilde{f}_{\nabla, \varphi} \quad \text { in }\right] 0, \bar{t}[, \\
v^{\prime}(0)=0, \quad v(\bar{t})=0 .
\end{array}\right.
$$

By a direct integration it is easy to show that

$$
v(t)=\int_{t}^{\bar{t}} \frac{d \sigma}{\beta a^{2}(\sigma)} \int_{0}^{\sigma} \beta a \tilde{f}_{\nabla, \varphi}(s) d s .
$$

Using hypothesis (H1) and proceeding as in Lemma 1, we can show that $v($.$) is$ nonnegative. Thus $\ell($.$) is nonnegative.$

Now let us set

$$
\mathcal{W}(x)= \begin{cases}\ell(\varphi(x)) & \text { if } x \in B(\bar{t}), \\ 0 & \text { if } x \in \Omega \backslash B(\bar{t}) .\end{cases}
$$

As already seen in part 3 (radial coordinates), $\mathcal{W}$ is a good candidate to verify:

$$
0 \leqslant \mathcal{W}(x)<u(x) \text { in } \Omega
$$

But to obtain (24) we have to use $\ell^{\prime} \circ \varphi$ and $\ell^{\prime \prime} \circ \varphi$ which are not necessarly defined. So we have to regularize Eqs. (20), (22) and (23). For this we need some approximation results.

Step 2. (A troncature result.)

LEMMA 3 (Troncature). - Let $f$ be a function defined on $\Omega$, bounded from below. For any $m \in \mathbb{R}$, we set

$$
f_{m}(x)=\inf (f(x), m)=[f(x)+m-|f(x)-m|], \quad \forall x \in \Omega .
$$

Then we have:

(i) $\left(f_{\varphi}\right)_{m}(t) \leqslant\left(f_{m}\right)_{\varphi}(t) \leqslant f_{\varphi}(t) \forall t$;

(ii) $\left(f_{\varphi}\right)_{m}(\varphi(x)) \leqslant\left(f_{m}\right)_{\varphi}(\varphi(x)) \leqslant f_{m}(x) \leqslant f(x) \forall x \in \Omega$, where we have set

$$
g_{m}(t)=\inf (g(t), m) \quad \forall t, \text { for any function } g(.) .
$$

Proof of Lemma 3. - First let us remark that for any function $k($.$) defined on [0, T]$ we have

$$
\widetilde{g}_{\varphi}(t)=k(t) \quad \forall t \in[0, T],
$$

if

$$
\widetilde{g}(x)=k \circ \varphi(x), \quad x \in \Omega .
$$

For any $m \in \mathbb{R}$, we have

$$
\left(f_{m}\right)_{\varphi}(t) \leqslant f_{\varphi}(t) \quad \forall t \in[0, T] .
$$


Let us prove

$$
\left(f_{\varphi}\right)_{m}(t) \leqslant\left(f_{m}\right)_{\varphi}(t) \quad \forall t
$$

For this let us set

$$
\begin{aligned}
& g(x)=f_{\varphi} \circ \varphi(x)=f_{\varphi}(\varphi(x)), \\
& g_{m}(x)=\frac{1}{2}\left[f_{\varphi}(\varphi(x))+m-\left|f_{\varphi}(\varphi(x))-m\right|\right], \\
& h(t)=\left(f_{\varphi}\right)_{m}(t)=\frac{1}{2}\left[f_{\varphi}(t)+m-\left|f_{\varphi}(t)-m\right|\right] .
\end{aligned}
$$

It is clear that

$$
h \circ \varphi(x)=h(\varphi(x))=g_{m}(x) \quad \forall x \in \Omega,
$$

which means from (25)

$$
\left(g_{m}\right)_{\varphi}(t)=h(t) \quad \forall t \in[0, T] .
$$

But

$$
g_{m}(x)=\inf \left(f_{\varphi}(\varphi(x)), m\right) \leqslant \inf (f(x), m)=f_{m}(x)
$$

since $f_{\varphi}(\varphi(x)) \leqslant f(x)$, or again

$$
g_{m}(x)=\left(f_{\varphi}\right)_{m}(\varphi(x)) \leqslant f_{m}(x) \leqslant f(x)
$$

Thus using (28) we obtain the result

$$
\left(g_{m}\right)_{\varphi}(t)=\left(f_{\varphi}\right)_{m}(t) \leqslant\left(f_{m}\right)_{\varphi}(t) \leqslant f_{\varphi}(t) \quad \forall t
$$

since $\left(k_{1}\right)_{\varphi} \leqslant\left(k_{2}\right)_{\varphi}$ if $k_{1} \leqslant k_{2}$. To prove (ii) it suffices to take $t=\varphi(x)$ in (30). H1)

Step 3. (Regularization of Eq. (22).) In a first step we can suppose that we have (cf.

$$
\int_{0}^{\bar{t}}\left[\int_{0}^{\sigma} a(s) \widetilde{f}_{\nabla, \varphi}(s) \beta(s) d s\right] \frac{d \sigma}{a^{2}(\sigma) \beta(\sigma)}>0 .
$$

The case where the left hand side of (31) is equal to zero will be treated later. From (31) there exists $m_{0}>0$ such that

$$
\forall m \geqslant m_{0} \int_{0}^{\bar{t}}\left[\int_{0}^{\sigma} a(s)\left(\widetilde{f}_{\nabla, \varphi}\right)_{m}(s) \beta(s) d s\right] \frac{d \sigma}{a^{2}(\sigma) \beta(\sigma)}>0 .
$$

In the sequel $m$ is fixed. Let $\rho_{\varepsilon}$ be the classical regular mollifier and let us define

$$
\begin{aligned}
& g_{\varepsilon}(t)=\left(\tilde{f}_{\nabla, \varphi}\right)_{m} * \rho_{\varepsilon}(t) \quad \forall t \in \mathbb{R}, \\
& \gamma_{\varepsilon}^{\varphi}(t)=\gamma^{\varphi} * \rho_{\varepsilon}(t) \quad \forall t \in \mathbb{R},
\end{aligned}
$$


after extending $\left(\widetilde{f}_{\nabla, \varphi}\right)_{m}$ and $\gamma^{\varphi}$ by zero to the all space $\mathbb{R}$. The operator star stands for the convolution operator. From Remark 2.1 it is not restrictive to assume $\gamma_{\varepsilon}^{\varphi}$ nonnegative in some neighbourhood of origin 0 . Thus we can apply Appendix B with $\gamma_{\varepsilon}^{\varphi}$ and conclude that the solution $a_{\varepsilon}$ of regularized equation of (20), is positive.

Now let us consider the solution $\ell_{\varepsilon}($.$) of the regularized equation of (22):$

$$
\left\{\begin{array}{l}
-\ell_{\varepsilon}^{\prime \prime}(t)-\frac{(\beta+\varepsilon)^{\prime}(t)}{(\beta+\varepsilon)(t)} \cdot \ell_{\varepsilon}^{\prime}(t)+\gamma_{\varepsilon}^{\varphi}(t) \ell_{\varepsilon}(t)=g_{\varepsilon}(t), \\
\ell_{\varepsilon}^{\prime}(0)=\ell_{\varepsilon}(\bar{t})=0 .
\end{array}\right.
$$

$\ell_{\varepsilon}($.$) is regular and its representation formula is$

$$
\ell_{\varepsilon}(t)=a_{\varepsilon}(t) . \int_{t}^{\bar{t}}\left[\int_{0}^{\sigma} a_{\varepsilon}(s) g_{\varepsilon}(s)(\beta+\varepsilon)(s) d s\right] \frac{d \sigma}{a_{\varepsilon}^{2}(\sigma) \cdot(\beta+\varepsilon)},
$$

$a_{\varepsilon}$ is solution of the regularized equation of (20). For any $t>0$

$$
\frac{\beta^{\prime}(t)}{\beta(t)} \cdot \ell_{\varepsilon}^{\prime}(t)=-\frac{\beta^{\prime}(t)}{\beta(t)} \cdot \int_{0}^{t} g_{\varepsilon} a_{\varepsilon} \cdot(\beta+\varepsilon) d s \times \frac{1}{a_{\varepsilon}^{2} \cdot(\beta+\varepsilon)(t)} .
$$

As $\beta($.$) is nondecreasing$

$$
\left|\frac{\beta^{\prime}(t)}{\beta(t)} \cdot \ell_{\varepsilon}^{\prime}(t)\right| \leqslant \frac{\beta^{\prime}(t)}{\beta(t)} \cdot \int_{0}^{t} a_{\varepsilon} \cdot\left|g_{\varepsilon}\right| d s \frac{1}{a_{\varepsilon}^{2}(t)} .
$$

But we have

$$
\left\|g_{\varepsilon}\right\|_{L^{\infty}(0, \bar{t})} \leqslant c, \quad\left\|a_{\varepsilon}\right\|_{L^{\infty}(0, \bar{t})} \leqslant c
$$

and $a_{\varepsilon}(x)>\inf [a(x) / x \in[0, \bar{t}]] / 2>0$, for $\forall \varepsilon \leqslant \varepsilon_{0}$, with $\varepsilon_{0}$ small enough, because $a_{\varepsilon} \rightarrow a$ uniformly. So

$$
\left|\frac{\beta^{\prime}(t)}{\beta(t)} \ell_{\varepsilon}^{\prime}(t)\right| \leqslant c \cdot \frac{\beta^{\prime}(t)}{\beta(t)} \int_{0}^{t} d s=c . t \frac{\beta^{\prime}(t)}{\beta(t)} \leqslant c, \quad \forall t .
$$

From (33) we deduce

$$
\begin{gathered}
\left|\ell_{\varepsilon}^{\prime}(t)\right| \leqslant c, \quad \forall t, \\
0 \leqslant \frac{\beta^{\prime}(t)}{\beta(t)}-\frac{\beta^{\prime}(t)}{\beta(t)+\varepsilon}=\frac{\varepsilon \beta^{\prime}(t)}{\beta(t) .(\beta(t)+\varepsilon)} \leqslant \frac{\beta^{\prime}(t)}{\beta(t)} .
\end{gathered}
$$

Using (33) we obtain

$$
\left|\left(\frac{\beta^{\prime}(t)}{\beta(t)}-\frac{\beta^{\prime}(t)}{\beta(t)+\varepsilon}\right) \ell_{\varepsilon}^{\prime}(t)\right| \leqslant c \quad \forall t .
$$


Let us take $t=\varphi(x)$ in (22), we obtain:

$$
\begin{aligned}
& -\ell_{\varepsilon}^{\prime \prime}(\varphi(x))-\frac{\beta^{\prime}(\varphi(x)) \ell_{\varepsilon}^{\prime}(\varphi(x))}{\beta(\varphi(x))}+\frac{\varepsilon \beta^{\prime}(\varphi(x))}{\beta(\varphi(x)) \cdot(\beta(\varphi(x))+\varepsilon)} \cdot \ell_{\varepsilon}^{\prime}(\varphi(x)) \\
& +\gamma_{\varepsilon}^{\varphi}(\varphi(x)) \ell_{\varepsilon}(\varphi(x))=: g_{\varepsilon}(\varphi(x)), \quad \forall x \in B(\bar{t}) .
\end{aligned}
$$

But let us recall that we have the following important relation

$$
\frac{\beta^{\prime}(\varphi(x))}{\beta(\varphi(x))}=\frac{\Delta \varphi(x)}{|\nabla \varphi(x)|^{2}}
$$

$\forall x \in \Omega \backslash\{x \in \Omega \mid \nabla \varphi(x)=0\}$, i.e., a.e. $x \in \Omega$. From (35) and (36) we deduce that

$$
\begin{aligned}
& -\Delta \ell_{\varepsilon}(\varphi)+\gamma_{\varepsilon}^{\varphi}(\varphi) \cdot|\nabla \varphi|^{2} \ell_{\varepsilon}(\varphi) \\
& \quad+\varepsilon \frac{\beta^{\prime}(\varphi) \cdot|\nabla \varphi|^{2}}{\beta(\varphi) \cdot(\beta(\varphi)+\varepsilon)} \ell_{\varepsilon}^{\prime}(\varphi)=g_{\varepsilon}(\varphi) \cdot|\nabla \varphi|^{2} \quad \text { a.e. } x \in B(\bar{t}) .
\end{aligned}
$$

Before to conclude we need to pass to the limit in (37) as $\varepsilon$ goes to zero.

Let us consider the function

$$
\psi_{\varepsilon}(x)=\varepsilon \frac{\beta^{\prime}(\varphi(x)) \cdot|\nabla \varphi|^{2} \ell_{\varepsilon}^{\prime}(\varphi(x))}{\beta(\varphi(x))(\beta(\varphi(x))+\varepsilon)}
$$

We have

$$
\psi_{\varepsilon}(x) \longrightarrow 0 \quad \forall x \in B(\bar{t}) \backslash\{x|| \nabla \varphi(x) \mid=0\}
$$

i.e., a.e. $x \in B(\bar{t})$, and $\forall x \in B(\bar{t})$ it follows

$$
\left|\psi_{\varepsilon}(x)\right| \leqslant|\nabla \varphi(x)|^{2} \cdot\left|\frac{\beta^{\prime}(\varphi(x))}{\beta(\varphi(x))} \ell_{\varepsilon}^{\prime}(\varphi(x))\right| \leqslant c
$$

by (33) and the regularity of $\varphi$ on $\bar{\Omega}$. So $\psi_{\varepsilon}$ tends strongly to zero in $L^{2}(\Omega)$ thanks to Lebesgue theorem. It is easy to see that $\gamma_{\varepsilon}^{\varphi}(\varphi) \cdot|\nabla \varphi|^{2} \ell_{\varepsilon}(\varphi)$ converges strongly to $\gamma^{\varphi}(\varphi)|\nabla \varphi|^{2} . \ell(\varphi)$ in $L^{2}(\Omega)$. On the other hand

$$
\left|\nabla \ell_{\varepsilon}(\varphi)\right|=\left|\ell_{\varepsilon}^{\prime}(\varphi) . \nabla \varphi\right| \leqslant c \quad \forall x \in B(\bar{t})
$$

using (33.1); finally $g_{\varepsilon}(\varphi)$ tends to $g(\varphi)$ as $\varepsilon$ goes to zero. Now we are able to pass to the limit in (37) as $\varepsilon$ goes to zero. We obtain

$$
\left\{\begin{array}{l}
-\Delta \ell(\varphi)+\gamma^{\varphi}(\varphi) \cdot \ell(\varphi) \cdot|\nabla \varphi|^{2}=g(\varphi)=\left(\tilde{f}_{\nabla, \varphi}\right)_{m}(\varphi) \cdot|\nabla \varphi|^{2} \quad \text { in } B(\bar{t}), \\
\left.\ell(\varphi)\right|_{\partial B(\bar{t})}=0, \quad|\nabla \ell(\varphi)| \leqslant c .
\end{array}\right.
$$

Let us estimate from above the right hand side of (38) and let us estimate from below its left hand side; we find

$$
\begin{cases}-\Delta \ell(\varphi)+c(x) \cdot \ell(\varphi) \leqslant f(x) & \text { in } B(\bar{t}) \\ \left.\ell(\varphi)\right|_{\partial B(\bar{t})}=0, \quad|\nabla \ell(\varphi)| \leqslant c & \text { in } B(\bar{t})\end{cases}
$$


since we have:

$$
c(x) \leqslant \gamma^{\varphi}(\varphi(x)) \cdot|\nabla \varphi(x)|^{2} \quad \text { by definition of } \gamma^{\varphi},
$$

and

$$
\left(\tilde{f}_{\nabla, \varphi}\right)_{m}(\varphi) \leqslant \frac{\widetilde{f}}{|\nabla \varphi|^{2}} \leqslant \frac{f}{|\nabla \varphi|^{2}} \quad \text { in } B(\bar{t}),
$$

from Lemma 3. Using the extension lemma of Appendix C, we can show, in the same way as in the last part of the proof of Theorem 1, that the solution $u$ of (1) is positive in $\Omega$. The first part of the theorem is so proved.

Step 4. Now it remains to examine the following case:

$$
\int_{0}^{\bar{t}}\left[\int_{0}^{\sigma} a(s) \tilde{f}_{\nabla, \varphi}(s) \beta(s) d s\right] \frac{d \sigma}{a^{2}(\sigma) \beta(\sigma)}=0
$$

(cf. (31)). If we have (39) let us set, for any $\varepsilon>0$

$$
h^{\varepsilon}(x)=f(x)+\varepsilon \quad \forall x \in B(\bar{t}) \backslash B\left(t_{0}\right) ; \quad h^{\varepsilon}(x)=f(x) \quad \forall x \in B\left(t_{0}\right) .
$$

For any $\varepsilon>0$, it is easy to see that we have:

$$
\int_{0}^{\bar{t}}\left[\int_{0}^{\sigma} a(s)\left(\widetilde{h^{\varepsilon}}\right)_{\nabla, \varphi}(s) \beta(s) d s\right] \frac{d \sigma}{a^{2}(\sigma)(\beta(\sigma)}>0 .
$$

We have to consider two cases:

(i) $B(\bar{t}) \subset \Omega$ and $f \not \equiv 0$ on $\Omega \backslash B(\bar{t})$.

Let $v$ be the solution of

$$
\left\{\begin{array}{l}
-\Delta v+c(x) \cdot v=k \quad \text { on } \Omega \\
\left.v\right|_{\partial \Omega}=0
\end{array}\right.
$$

where

$$
k(x)= \begin{cases}f(x) & \forall x \in B(\bar{t}) \\ 0 & \forall x \in \Omega / B(\bar{t}) .\end{cases}
$$

Then we apply the first part of the proof to obtain

$$
v_{\varepsilon}(x)>0 \quad \text { a.e. } x \in \Omega,
$$

where $v_{\varepsilon}$ is the solution of

$$
\left\{\begin{array}{l}
-\Delta v_{\varepsilon}+c \cdot v_{\varepsilon}=k^{\varepsilon} \quad \text { on } \Omega \\
\left.v_{\varepsilon}\right|_{\partial \Omega}=0
\end{array}\right.
$$

with $k^{\varepsilon}$ is defined similarly to $h^{\varepsilon}$ given above. Let us take the limit in (42) as $\varepsilon$ goes to zero. We obtain 


$$
\begin{aligned}
& v_{\varepsilon} \rightarrow v \text { in } H_{0}^{1}(\Omega) \text { weakly, } \\
& v(x) \geqslant 0 \quad \text { a.e. } x \in \Omega .
\end{aligned}
$$

As from the strong maximum principle we have

$$
u(x)>v(x) \text { a.e. } x \in \Omega,
$$

the result is then clearly proved.

(ii) $B(\bar{t})=\Omega$ or $B(\bar{t}) \subset \Omega$ with $f \equiv 0$ on $\Omega \backslash B(\bar{t})$. Here we also apply the first part of the proof to obtain

$$
u_{\varepsilon}(x)>0 \text { a.e. } x \in \Omega,
$$

where $u_{\varepsilon}$ is the solution of (1) with the right hand side $f$ replaced by $h^{\varepsilon}$. To achieve the proof of our result, we pass to the limit as $\varepsilon$ goes to zero. We finally obtain

$$
u_{\varepsilon} \rightarrow u \quad \text { in } H_{0}^{1}(\Omega) \text { weakly }
$$

and

$$
u(x) \geqslant 0 \quad \text { a.e. } x \in \Omega .
$$

Now the proof is achieved.

\section{General shape's function}

In order to improve our results, we are now going to enclose the negative support of $f$ by an increasing family of level sets of some shape function $\varphi$ satisfying the inclusion:

$$
E_{f} \subseteq B\left(t_{0}\right) \subset B(t) \subseteq \Omega \quad \forall t, t_{0}<t \leqslant T .
$$

We would like to choose $\varphi$ in such a way that the measures of the sets $\left[B\left(t_{0}\right) \backslash E_{f}\right]$ and $[\Omega \backslash B(T)]$ are as small as possible. The smaller these measures are, the better these results. Indeed in the previous sections, we were not able to take into account the behaviour of $f$ in these two areas. We shall see how to use shape functions in general to overcome this difficulty.

Let $\varphi$ be a regular function defined on $\Omega$ such that

$$
\left.\varphi\right|_{\partial \Omega}=\sup \{\varphi(x) \mid x \in \bar{\Omega}\}=T .
$$

Since we can replace $\varphi$ by $\varphi-\inf [\varphi(x) \mid x \in \bar{\Omega}]$ if necessary, we can always assume that $\varphi$ is nonnegative in $\Omega$. We suppose that, for any $t, 0<t<T$, the level sets of $\varphi$

$$
B(t)=\{x \in \Omega \mid \varphi(x)<t\}
$$

are connected and regular, i.e., the boundaries $\partial B(t)$ are regular. The sets $B(t)$ are an increasing family of open subsets of $\Omega$. In ellipsoïdal coordinates, $\varphi$ satisfies an important relation of structure given by (19.1) proved in Lemma 3. For general shape's function we do not have (19.1). So we are obliged to assume the following hypothesis: 


$$
\begin{aligned}
& \left.\left.\hat{\beta}_{\varphi}(t)=\frac{\alpha_{1}}{t}+\delta_{1}(t) \quad \forall t \in\right] 0, T\right], \\
& \left.\left.\hat{\beta}^{\varphi}(t)=\frac{\alpha_{2}}{t}+\delta_{2}(t) \quad \forall t \in\right] 0, T\right],
\end{aligned}
$$

where

$$
\hat{\beta}(x)=\Delta \varphi(x) /|\nabla \varphi(x)|^{2},
$$

$\alpha_{i}$ are positive constants, $0<\alpha_{1} \leqslant \alpha_{2}$, and $\delta_{i}$ are bounded positive functions defined on $[0, T], i=1,2$. Let us set

$$
\beta_{i}(t)=t^{\alpha_{i}} \exp \left[\int_{0}^{t} \delta_{i}(s) d s\right], \quad i=1,2,
$$

and define, for any $\sigma \in[0, T]$

$$
\check{\beta}_{\sigma}(s)= \begin{cases}\beta_{1}(s) & \text { if } s \in[0, \sigma], \\ \beta_{2}(s) & \text { if } s \in[\sigma, T],\end{cases}
$$

and consider the application $\Lambda$

$$
\sigma \in[0, T] \rightarrow \Lambda(\sigma)=\int_{0}^{\sigma} \widetilde{f}_{\varphi}(s) a_{\sigma}(s) \beta_{\sigma}(s) d s,
$$

where, for any $\sigma \in[0, T], a_{\sigma}($.$) denotes the solution of:$

$$
\left\{\begin{array}{l}
\left.-\frac{1}{\ddot{\beta}_{\sigma}}\left(\check{\beta}_{\sigma} a_{\sigma}^{\prime}\right)^{\prime}+\gamma^{\varphi} \cdot a_{\sigma}=0 \quad \text { on }\right] 0, T[, \\
a_{\sigma}^{\prime}(0)=0, \quad a_{\sigma}(T)=1 .
\end{array}\right.
$$

In this section we also assume the localization hypothesis (H0). Before introducing the hypothesis needed in this part, let us give the following results

PROPOSITION 2. - We have the following estimate

$$
\left\|a_{\sigma}\right\|_{W^{1, \infty}([0, T[)} \leqslant c
$$

where $c$ is a constant independant of $\sigma$.

Its proof is an immediate consequence of the proposition of Appendix B.

PROPOSITION $3($ Continuity with respect to the parameter $\sigma)$. The function $a(\sigma,)=$. $a_{\sigma}($.$) satisfies$

(i) $\left\|a_{\sigma_{1}}-a_{\sigma_{2}}\right\|_{H^{1}(0, T[)} \leqslant c .\left|\sigma_{1}-\sigma_{2}\right|$, where $c$ is a constant independant of $\beta_{\sigma_{1}}$ and $\beta_{\sigma_{2}}$,

(ii) $\left\|a_{\sigma_{1}}-a_{\sigma_{2}}\right\|_{L^{\infty}([0, T])} \leqslant c .\left|\sigma_{1}-\sigma_{2}\right|$, where $c$ is a constant independant of $\beta_{\sigma_{1}}$ and $\beta_{\sigma_{2}}$,

(iii) $\left|a(\sigma, t)-a\left(\sigma^{\prime}, t^{\prime}\right)\right| \leqslant c\left[\left|\sigma_{1}-\sigma_{2}\right|+\left|t-t^{\prime}\right|\right]$ where $c$ is a constant. 
To be more clear, this result will be proved later. As in Section 5, we propose to construct a function $\ell$ solution of Eq. (22) for a suitable $\theta$ and such that $\ell \circ \varphi$ is a positive subsolution of Eq. (1). To guarantee the positivity of $\ell$ we need hypothesis (H3). Hypothesis (H4) allows us to state that $\ell \circ \varphi$ is a subsolution of (1). To state our hypothesis we need some preliminaries.

Let us set

$$
\begin{gathered}
\bar{a}(t)= \begin{cases}\max \left[a_{\sigma}(t) \mid \sigma, t_{0} \leqslant \sigma \leqslant T\right], & 0 \leqslant t \leqslant t_{0}, \\
\min \left[a_{\sigma}(t) \mid \sigma, t_{0} \leqslant \sigma \leqslant T\right], & t_{0} \leqslant t \leqslant T,\end{cases} \\
\underline{a}(t)= \begin{cases}\min \left[a_{\sigma}(t) \mid \sigma, t_{0} \leqslant \sigma \leqslant T\right], & 0 \leqslant t \leqslant t_{0}, \\
\max \left[a_{\sigma}(t) \mid \sigma, t_{0} \leqslant \sigma \leqslant T\right], & t_{0} \leqslant t \leqslant T,\end{cases} \\
\bar{\beta}(t)= \begin{cases}\beta_{1}(t), & 0 \leqslant t \leqslant t_{0}, \\
\beta_{1}(t), & t_{0} \leqslant t \leqslant T,\end{cases} \\
\underline{\beta}(t)= \begin{cases}\beta_{1}(t), & 0 \leqslant t \leqslant t_{0}, \\
\beta_{2}(t), & t_{0} \leqslant t \leqslant T .\end{cases}
\end{gathered}
$$

From Proposition 3 we have

$$
1 \geqslant \min _{\sigma \geqslant t_{0}} a_{\sigma}(t) \geqslant \min _{\substack{\sigma \geqslant t_{0} \\ t \geqslant 0}} a(\sigma, t)>0
$$

since $a(\sigma, t)>0 \forall \sigma \geqslant 0, \forall t \geqslant 0$, by the maximum principle for Eq. (45) (cf. Appendix B). In the same way

$$
1 \geqslant \max _{\sigma \geqslant t_{0}} a_{\sigma}(t) \geqslant \min _{\substack{\sigma \geqslant t_{0} \\ t \geqslant 0}} a(\sigma, t)>0 .
$$

(H3) There exists $\bar{t}, t_{0}<\bar{t} \leqslant T$ such that

$$
\int_{0}^{\bar{t}} \frac{1}{\underline{a}^{2}(t) \underline{\beta}(t)}\left[\int_{0}^{s} \bar{a}(\theta) \bar{\beta}(\theta) \tilde{f}_{\nabla, \varphi}(\theta) d \theta\right] d s>0 .
$$

Comments about (H3). - In the ellipsoïdal coordinates (H3) is identical to (H1) since $\beta_{\sigma}=\beta_{1}=\beta_{2} \forall \sigma$.

(H4) $\forall t, t_{0} \leqslant t \leqslant \bar{t}$

$$
\tilde{f}_{\nabla, \varphi}-\gamma^{\varphi}(t) . \int_{t}^{\bar{t}} d s \int_{t_{0}}^{s} \tilde{f}_{\nabla, \varphi}(\theta) d \theta>0 .
$$

Comments about (H4). - This hypothesis establishes the quantitative link between $\tilde{f}_{\nabla, \varphi}^{+}, \tilde{f}_{\nabla, \varphi}^{-}$and their supports. Let us recall that $\gamma^{\varphi}(t)=\left(c^{+} /|\nabla \varphi|\right)^{\varphi}(t)$. So if $c(x) \leqslant 0$ in $\Omega \backslash B\left(t_{0}\right)$, (H4) is obviously satisfied. 
Let us set $\psi(t)=\int_{t}^{\bar{t}} \int_{t_{0}}^{s} \tilde{f}_{\nabla, \varphi}(\theta) d \theta$. It is easy to see that $\psi(t) \geqslant 0$ in $] t_{0}, \bar{t}[$, and

$$
\left\{\begin{array}{l}
\left.-\psi^{\prime \prime}(t)-\gamma^{\varphi}(t) . \psi(t)>0 \quad \text { in }\right] t_{0}, \bar{t}[, \\
\psi(\bar{t})=\psi^{\prime}\left(t_{0}\right)=0 .
\end{array}\right.
$$

From (H2), $\left\|\gamma^{\varphi}\right\|_{\infty}$ satisfies $\left\|\gamma^{\varphi}\right\|_{\infty}<\frac{\pi}{\sqrt{2}} \frac{1}{\bar{t}^{2}}$. Thus it verifies also $\left\|\gamma^{\varphi}\right\|_{\infty}<\left(\frac{\pi}{2}\right)^{2} \frac{1}{\left(\bar{t}-t_{0}\right)^{2}}$ which entails that the operator $-\frac{d^{2}}{d t^{2}}-\gamma^{\varphi}(t) . I$ verifies the maximum principle in $] t_{0}, \bar{t}[$. Thus if we give a nonpositive function $g_{1}$ in $B\left(t_{0}\right)=\left\{x \in \Omega \mid \varphi(x)<t_{0}\right\}$ from (46), we can see that $(\mathrm{H} 4)$ permits us to construct a very large class of nonnegative functions $g_{2}$ in $\Omega \backslash B\left(t_{0}\right)$ such that Eq. (1) has a positive solution if

$$
f(x)= \begin{cases}g_{1}(x) & \text { in } B\left(t_{0}\right), \\ g_{2}(x) & \text { in } \Omega \backslash B\left(t_{0}\right) .\end{cases}
$$

Indeed let $h(t)$ be a continuous function nonnegative in $\left[t_{0}, \bar{t}\right]$ and let us consider the solution $\psi$ of

$$
\left\{\begin{array}{l}
-\psi^{\prime \prime}-\gamma^{\varphi} \cdot \psi=h \quad \text { in }\left[t_{0}, \bar{t}\right] \\
\psi^{\prime}\left(t_{0}\right)=\psi(\bar{t})=0
\end{array}\right.
$$

$\psi$ is positive and $-\psi^{\prime \prime}$ is nonnegative and continuous. For any $\lambda \in \mathbb{R}, \lambda>0$ set

$$
f_{\nabla, \varphi}(t):= \begin{cases}\left(g_{1}\right)_{\varphi}(t) & \text { in }\left[0, t_{0}\right], \\ -\lambda \psi^{\prime \prime}(t) & \text { in }\left[t_{0}, \bar{t}\right] .\end{cases}
$$

There exists $\lambda_{0}>0$ such that, for any $\lambda \geqslant \lambda_{0}$, the function $f_{\nabla, \varphi}$ satisfies (H3). Then the suitable function $f$ is

$$
f(x)= \begin{cases}f_{\nabla, \varphi} \circ \varphi(x) \cdot|\nabla \varphi(x)|^{2} \quad \text { in } B(\bar{t}), \\ 0, \quad \text { for instance in } \Omega \backslash B(\bar{t}) .\end{cases}
$$

Now let us consider the following illustrative example:

$$
f(x)= \begin{cases}-a \cdot|\nabla \varphi(x)|^{2} & \text { in } B\left(t_{0}\right), a>0, \\ b \cdot|\nabla \varphi(x)|^{2} & \text { in } \Omega \backslash B\left(t_{0}\right), b>0 .\end{cases}
$$

In this case (H4) means

$$
\left\{\begin{array}{l}
0 \leqslant \gamma^{\varphi}(t) \leqslant \frac{2 b}{b\left(\bar{t}-t_{0}\right)^{2}-b\left(t-t_{0}\right)^{2}-2 a \int_{0}^{t_{0}} \beta_{1}(s) d s \cdot \int_{t}^{\bar{t}} \frac{d s}{\beta_{2}(s)}} \\
\forall t \geqslant t_{0} \text { such that } b\left(\bar{t}-t_{0}\right)^{2}>b\left(t-t_{0}\right)^{2}+2 a \cdot \int_{0}^{t_{0}} \beta_{1} d s \cdot \int_{t}^{\bar{t}} \frac{d s}{\beta_{2}} .
\end{array}\right.
$$

And (47) is obviously satisfied since by (H2) we have

$$
\left\|\gamma^{\varphi}\right\|_{\infty}<\frac{\pi}{\sqrt{2}} \frac{1}{\bar{t}^{2}}
$$

that is to say, $\left\|\gamma^{\varphi}\right\|_{\infty}<2 /\left(\bar{t}-t_{0}\right)^{2}$ if for instance $t_{0}>(1-\sqrt{2 \sqrt{2} / \pi}) \bar{t}$. 
Proposition 4. - Suppose (H4) and (H0). Then we have: $\forall \sigma \in\left[t_{0}, \bar{t}\right]$ the solution $\ell_{\sigma}($.) of Eq. (22) is positive in $] 0, \bar{t}[$.

Proof. - From the representation formula of $v_{\sigma}$

$$
\ell_{\sigma}(t)=a_{\sigma}(t) \cdot v_{\sigma}(t)=a_{\sigma}(t) \cdot \int_{t}^{\bar{t}} \frac{d s}{a_{\sigma}^{2}(s) \beta_{\sigma}(s)} \int_{0}^{s} a_{\sigma}(\theta) \beta_{\sigma}(\theta) \widetilde{f}_{\nabla, \varphi}(\theta) d \theta .
$$

From (H4) it is easy to see that

$$
v_{\sigma}(0) \geqslant \int_{0}^{\bar{t}} \frac{1}{\underline{a}^{2}} \underline{\beta}\left[\int_{0}^{s} \bar{a} \bar{\beta} \widetilde{f}_{\nabla, \varphi} d \theta\right] d s>0
$$

which implies that $v_{\sigma}(t)>0, \forall t \in\left[0, t_{0}\right]$, using the localization hypothesis. To achieve the proof we apply the maximum principle to Eq. (23) in $] t_{0}, \bar{t}$ [ and we deduce that $v_{\sigma}(t)>0, \forall t \in\left[t_{0}, \bar{t}\left[\right.\right.$, that is to say $\ell_{\sigma}($.$\left.) is positive in \right] 0, \bar{t}[$.

Proposition 5. - The function $\ell_{\sigma}(t)=\ell(\sigma, t)$ solution of $(22)$ in $[0, \bar{t}]$ verifies: $\forall \sigma_{0}$, $0<\sigma_{0}<t_{0}, \exists c=c\left(\sigma_{0}\right)>0$ such that

$$
\left\{\begin{array}{l}
\left|\ell(\sigma, t)-\ell\left(\sigma^{\prime}, t^{\prime}\right)\right| \leqslant c\left[\left|\sigma-\sigma^{\prime}\right|+\left|t-t^{\prime}\right|\right] \\
\forall \sigma, \sigma^{\prime} \in\left[\sigma_{0}, \bar{t}\right] \forall t, t^{\prime} \in\left[\sigma_{0}, \bar{t}\right]
\end{array}\right.
$$

Proof. - It sufficies to state that $v_{\sigma}(t)=v(\sigma, t)$ verifies (48). For this we use Proposition 3 and we proceed as in its proof. Let us give $\sigma_{0}<\sigma<\sigma^{\prime}, 0 \leqslant t<t^{\prime} \leqslant \bar{t}$; we have

$$
\begin{gathered}
v(\sigma, t)-v\left(\sigma^{\prime}, t^{\prime}\right)=v(\sigma, t)-v\left(\sigma^{\prime}, t\right)+v\left(\sigma^{\prime}, t\right)-v\left(\sigma^{\prime}, t^{\prime}\right), \\
\left|v\left(\sigma^{\prime}, t\right)-v\left(\sigma^{\prime}, t^{\prime}\right)\right| \leqslant \int_{t}^{t^{\prime}} \frac{d s}{a_{\sigma^{\prime}}^{2} \beta_{\sigma^{\prime}}}\left(\int_{0}^{s} a_{\sigma^{\prime}} \beta_{\sigma^{\prime}}\left|\tilde{f}_{\nabla, \varphi}\right| d \theta\right) \\
\leqslant \int_{t}^{t^{\prime}} \frac{d s}{a_{\sigma^{\prime}}^{2}} \int_{0}^{s} a_{\sigma^{\prime}}\left|\tilde{f}_{\nabla, \varphi}\right| d \theta
\end{gathered}
$$

that is

$$
\left|v\left(\sigma^{\prime}, t\right)-v\left(\sigma^{\prime}, t^{\prime}\right)\right| \leqslant c\left|t-t^{\prime}\right|,
$$

where $c$ is a constant, since we have

$$
\inf [a(\sigma, t) \mid(\sigma, t) \in[0, \bar{t}] \times[0, \bar{t}]]>0,
$$

and

$$
\sup [a(\sigma, t) \mid(\sigma, t) \in[0, \bar{t}] \times[0, \bar{t}]]<+\infty,
$$

from Proposition 3. The first term in the right hand side of (49) can be written: 


$$
\begin{aligned}
\left|v(\sigma, t)-v\left(\sigma^{\prime}, t\right)\right| \leqslant & \int_{t}^{\bar{t}} \frac{\left|a_{\sigma}^{2}-a_{\sigma^{\prime}}^{2}\right|}{a_{\sigma}^{2} a_{\sigma^{\prime}}^{2} \beta \sigma} \int_{0}^{s} a_{\sigma} \beta_{\sigma}\left|\widetilde{f}_{\nabla, \varphi}\right| d \theta d s \\
& +\int_{t}^{\bar{t}} \frac{\left|\beta_{\sigma}-\beta_{\sigma^{\prime}}\right|}{a_{\sigma^{\prime}}^{2} \beta_{\sigma} \beta_{\sigma^{\prime}}} \int_{0}^{s} a_{\sigma} \beta_{\sigma}\left|\widetilde{f}_{\nabla, \varphi}\right| d \theta d s \\
& +\int_{t}^{\bar{t}} \frac{1}{a_{\sigma^{\prime}}^{2} \beta_{\sigma^{\prime}}} \int_{0}^{s}\left[\left|a_{\sigma}-a_{\sigma^{\prime}}\right| \beta_{\sigma}+\left|\beta_{\sigma}-\beta_{\sigma^{\prime}}\right| a_{\sigma^{\prime}}\right]\left|\widetilde{f}_{\nabla, \varphi}\right| d \theta d s .
\end{aligned}
$$

Remarking that $\left|\beta_{\sigma}(s)-\beta_{\sigma^{\prime}}(s)\right|=\left|\beta_{1}(s)-\beta_{2}(s)\right| \cdot \chi_{\left[\sigma, \sigma^{\prime}\right]}(s)$, using (50), (51) and the results of Proposition 3, it is easy to state the final result.

Proposition 6. - Under hypothesis (H4), for any $\sigma \in[0, \bar{t}], \ell_{\sigma}($.$) satisfies:$

$$
\left.\gamma^{\varphi}(t) \ell_{\sigma}(t)-\widetilde{f}_{\nabla, \varphi}(t)<0 \quad \text { in }\right] t_{0}, \bar{t}[
$$

Proof. - From (22) we have

$$
\begin{aligned}
\ell_{\sigma}^{\prime}(t)= & \frac{1}{\check{\beta_{\sigma}}(t)} \int_{0}^{t} \check{\beta}_{\sigma}\left(\gamma^{\varphi} \ell_{\sigma}-\widetilde{f}_{\nabla, \varphi}\right)(s) d s, \\
0 \leqslant \ell_{\sigma}(t)= & \int_{t}^{\bar{t}} \frac{1}{\check{\beta}_{\sigma}(s)}\left[\int_{0}^{s} \check{\beta}_{\sigma}\left(\widetilde{f}_{\nabla, \varphi}-\gamma^{\varphi} \ell_{\sigma}\right) d \theta\right] d s, \\
0 \leqslant \ell_{\sigma}(t) \leqslant & \int_{t}^{\bar{t}} \frac{1}{\check{\beta}_{\sigma}(s)} \int_{0}^{t_{0}} \check{\beta}_{\sigma}\left(\widetilde{f}_{\nabla, \varphi}-\gamma^{\varphi} \ell_{\sigma}\right) d \theta d s \\
& +\int_{t}^{\bar{t}} \frac{1}{\check{\beta}_{\sigma}(s)} \int_{t_{0}}^{s} \check{\beta}_{\sigma}\left(\widetilde{f}_{\nabla, \varphi}-\gamma^{\varphi} \ell_{\sigma}\right) d \theta d s, \quad \forall t \geqslant t_{0}, \\
& \int_{\bar{t}} \frac{1}{\check{\beta}_{\sigma}(s)}\left(\int_{t_{0}}^{s} \check{\beta}_{\sigma} \widetilde{f}_{\nabla, \varphi} d \theta\right), \quad \forall t \geqslant t_{0},
\end{aligned}
$$

since $\gamma^{\varphi} \ell_{\sigma}$ is nonnegative, and $\widetilde{f}_{\nabla, \varphi}$ is nonpositive in $\left[0, t_{0}\right]$. And using the fact that $\check{\beta_{\sigma}}$ is nondecreasing, we obtain

$$
0 \leqslant \ell_{\sigma}(t) \leqslant \int_{t}^{\bar{t}} \int_{t_{0}}^{s} \widetilde{f}_{\nabla, \varphi}(\theta) d \theta
$$


From this it follows that

$$
\gamma^{\varphi}(t) \ell_{\sigma}(t)-\widetilde{f}_{\nabla, \varphi}(t)<\gamma^{\varphi}(t) \int_{t}^{\bar{t}} \int_{t_{0}}^{s} \widetilde{f}_{\nabla, \varphi}(\theta) d \theta-\widetilde{f}_{\nabla, \varphi}(t)<0 \quad \forall t \geqslant t_{0}
$$

by (H4).

We need the previous proposition to prove the following result:

PROPOSITION 7. - There exists $\hat{\sigma}>t_{0}$ such that $\ell_{\hat{\sigma}}($.$) verifies:$

$$
\begin{array}{ll}
\ell_{\hat{\sigma}}^{\prime}(t) \geqslant 0 & \forall t \in[0, \hat{\sigma}[, \\
\ell_{\hat{\sigma}}^{\prime}(t) \leqslant 0 & \forall t \in] \hat{\sigma}, \bar{t}[.
\end{array}
$$

Proof. - Let us set

$$
V(\sigma, t)=\check{\beta}_{\sigma}(t) \cdot \ell_{\sigma}^{\prime}(t) \quad \forall \sigma \in[0, \bar{t}], \forall t \neq \sigma .
$$

From Eq. (22) it follows

$$
V(\sigma, t)=\int_{0}^{t} \check{\beta}_{\sigma}\left(\gamma^{\varphi} \ell_{\sigma}-\tilde{f}_{\nabla, \varphi}\right) d \theta
$$

which shows that $t \rightarrow V(\sigma, t)$ is also continuous at $t=\sigma$.

First step. We claim that there exists $\sigma>t_{0}$ such that $V(\sigma, \sigma)>0$.

By Proposition 5 we have:

$$
V(\sigma, \sigma) \geqslant V\left(t_{0}, t_{0}\right)-2 c\left|\sigma-t_{0}\right| \quad \forall \sigma \geqslant t_{0}
$$

But $V\left(t_{0}, t_{0}\right)$ is positive by using the hypothesis of localization and since $\gamma^{\varphi}(t) \geqslant 0$. Thus

$$
V(\sigma, \sigma)>0 \quad \forall \sigma \text { such that } 0<\sigma-t_{0}<\frac{V\left(t_{0}, t_{0}\right)}{4 c} .
$$

Second step. We claim that $V(\bar{t}, \bar{t})<0$.

Consider

$$
V(\bar{t}, t)=\int_{0}^{t} \check{\beta}_{\bar{t}}\left(\gamma^{\varphi} \ell_{\bar{t}}-\widetilde{f}_{\nabla, \varphi}\right) d s .
$$

We have $\ell_{\bar{t}}(t)>0$ in $\left[0, \bar{t}\left[\right.\right.$. In addition $\ell_{\bar{t}}(\bar{t})=0$. So $\ell_{\bar{t}}($.) is nonincreasing in a neighbourhood $\mathcal{V}(\bar{t})$ of $(\bar{t})$, i.e., $V(\bar{t}, t) \leqslant 0, \forall t \in \mathcal{V}(\bar{t})$. We argue by contradiction. That is we suppose $V(\bar{t}, \bar{t})=0$. Let us recall that

$$
V(\bar{t}, t)>0 \quad \forall t \leqslant t_{0}
$$

We have two cases to consider. 
First case: $V(\bar{t}, t) \geqslant 0 \forall t, t_{0} \leqslant t \leqslant \bar{t}$ that is, using (55), $\ell_{\bar{t}}($.$) is nondecreasing on$ $[0, \bar{t}]$. As $\ell_{\bar{t}}(0)>0$ and $\ell_{\bar{t}}(\bar{t})=0$, we obtain a contradiction.

Second case: there exists $t \in] t_{0}, \bar{t}$ [ such that $V(\bar{t}, t)<0$. As from $(55) V\left(\bar{t}, t_{0}\right)>0$, we deduce that there exists $\hat{t} \in] t_{0}, \bar{t}$ [ such that $V(\bar{t}, \hat{t})=0$. Now from (53) we can write

$$
V(\bar{t}, \bar{t})-V(\bar{t}, \hat{t})=\int_{\hat{t}}^{\bar{t}} \check{\beta}_{\bar{t}}\left(\gamma^{\varphi} \ell_{\bar{t}}-\widetilde{f}_{\nabla, \varphi}\right) d s=0 .
$$

This is a contradiction since

$$
\left.\gamma^{\varphi} \ell_{\bar{t}}-\widetilde{f}_{\nabla, \varphi}<0 \quad \text { in }\right] t_{0}, \bar{t}[
$$

by using hypothesis (H4) and Proposition 6. Consequently $V(\bar{t}, \bar{t})<0$.

Third step. We claim that there exists $\hat{\sigma} \in] t_{0}, \bar{t}\left[\right.$ such that $V(\hat{\sigma}, \hat{\sigma})=0$ that is $\ell_{\hat{\sigma}}($.$) is$ derivable at $\hat{\sigma}$ and $\ell_{\hat{\sigma}}^{\prime}(\hat{\sigma})=0$.

Let us set

$$
\begin{aligned}
& \mathcal{W}=\{\sigma \in] t_{0}, \bar{t}[\mid V(\sigma, \sigma)>0\} \\
& \hat{\sigma}=\sup \{\sigma \mid \sigma \in \mathcal{W}\} .
\end{aligned}
$$

It is clear that $\mathcal{W} \neq \varnothing$ and $t_{0}<\hat{\sigma}<\bar{t}$. We claim that $V(\hat{\sigma}, \hat{\sigma})=0$. We argue by contradiction. Suppose $V(\hat{\sigma}, \hat{\sigma})>0$. From Proposition 5 we deduce

$$
V(\sigma, \sigma) \geqslant V(\hat{\sigma}, \hat{\sigma})-2 c|\sigma-\hat{\sigma}|, \quad \forall \sigma
$$

So $V(\sigma, \sigma)>0$ for any $\sigma$ such that $0<\sigma-\hat{\sigma}<V(\hat{\sigma}, \hat{\sigma}) /(4 c)$. This contradicts the definition of $\hat{\sigma}$. So the claim is proved.

Fourth step. Let us prove that $V(\hat{\sigma}, t)<0, \forall t \in] \hat{\sigma}, \bar{t}[$. Suppose that there exists $t \in] \hat{\sigma}, \bar{t}[$ such that $V(\hat{\sigma}, t)>0$. So there exists $\widetilde{t}, \hat{\sigma}<\widetilde{t}<\bar{t}$ such that $V(\hat{\sigma}, \widetilde{t})=0$. This and the claim of the third step give:

$$
V(\hat{\sigma}, \widetilde{t})-V(\hat{\sigma}, \hat{\sigma})=\int_{\hat{\sigma}}^{\tilde{t}} \check{\beta}_{\hat{\sigma}}\left(\gamma^{\varphi} \ell_{\hat{\sigma}}-\widetilde{f}_{\nabla, \varphi}\right) d \theta=0
$$

which contradicts hypothesis (H4) from Proposition 6. Consequently we have proved that:

$$
\left.\ell_{\hat{\sigma}}^{\prime}(\hat{\sigma})=0, \quad \ell_{\hat{\sigma}}^{\prime}(t) \geqslant 0 \quad \forall t \in\right] 0, \hat{\sigma}\left[, \quad \ell_{\hat{\sigma}}^{\prime}(t) \leqslant 0 \quad \forall t \in\right] \hat{\sigma}, \bar{t}[.
$$

Proof of Proposition 3. - It is very technical. To simplify let us set

$$
a_{i}=a_{\sigma_{i}}, \quad \check{\beta}_{\sigma_{i}}=v_{i}, \quad i=1,2 .
$$

From (45) we have

$$
a_{1}^{\prime}(t)-a_{2}^{\prime}(t)=\Delta_{1}(t)+\Delta_{2}(t)
$$


where

$$
\begin{aligned}
\Delta_{1}(t) & =\frac{1}{v_{1}(t)} \int_{0}^{t} v_{1} \cdot \gamma^{\varphi} \cdot\left(a_{1}-a_{2}\right) d s \\
\Delta_{2}(t) & =\frac{1}{v_{1}(t)} \cdot \int_{0}^{t} v_{1} \cdot \gamma^{\varphi} a_{2} d s-\frac{1}{v_{2}(t)} \cdot \int_{0}^{t} v_{2} \cdot \gamma^{\varphi} a_{2} d s \\
& =\int_{0}^{t} \frac{v_{2}(t) v_{1}(s)-v_{2}(s) v_{1}(t)}{v_{1}(t) v_{2}(t)} \cdot \gamma^{\varphi} \cdot a_{2} d s .
\end{aligned}
$$

For any $t \in[0, T]$ we have obviously

$$
\left|\int_{\sigma_{1}}^{\sigma_{2}} a_{2}(s) \gamma^{\varphi}(s) . \frac{\nu_{2}(t) v_{1}(s)-v_{2}(s) v_{1}(t)}{v_{1}(t) \nu_{2}(t)} d s\right| \leqslant 2 \int_{\sigma_{1}}^{\sigma_{2}} a_{2}(s)\left|\gamma^{\varphi}(s)\right| d s .
$$

To be more clear we can suppose $\sigma_{1}<\sigma_{2}$. From (57) and using Cauchy-Schwarz inequality we can see that

$$
\int_{0}^{T}\left|\Delta_{1}(t)\right|^{2} d t \leqslant\left\|\gamma^{\varphi}\right\|_{\infty}^{2} \cdot \frac{T^{2}}{2} \cdot\left\|a_{1}-a_{2}\right\|_{L^{2}}^{2}
$$

To estimate $\Delta_{2}$ we have to consider three cases:

First case. $t \in\left[0, \sigma_{1}\right], v_{1}(t)=\check{\beta}_{\sigma_{1}}(t)=\beta_{\sigma}(t)=\beta_{1}(t), v_{2}(t)=\check{\beta}_{\sigma_{2}}(t)=\beta_{1}$. So

$$
\Delta_{2}(t)=0 \quad \forall t \in\left[0, \sigma_{1}\right]
$$

Second case. $t \in] \sigma_{1}, \sigma_{2}\left[, v_{1}(t)=\check{\beta}_{\sigma_{1}}(t)=\beta_{2}(t), \nu_{2}(t)=\check{\beta}_{\sigma_{2}}(t)=\beta_{1}(t)\right.$. We have

$$
\Delta_{2}(t)=\int_{0}^{\sigma_{1}} E(t, s) d s+\int_{\sigma_{1}}^{t} E(t, s) d s
$$

where

$$
E(t, s)=\frac{v_{2}(t) v_{1}(s)-v_{2}(s) v_{1}(t)}{v_{1}(t) \cdot v_{2}(t)} \cdot a_{2}(s) \gamma^{\varphi}(s) .
$$

From the first case we have

$$
\int_{0}^{\sigma_{1}} E(t, s) d s=0
$$

and $\Delta_{2}(t)$ becomes

$$
\Delta_{2}(t)=\int_{\sigma_{1}}^{t} \frac{v_{2}(t) v_{1}(s)-v_{2}(s) v_{1}(t)}{v_{1}(t) \cdot v_{2}(t)} \cdot a_{2}(s) \gamma^{\varphi}(s) d s
$$




$$
\left|\Delta_{2}(t)\right| \leqslant \int_{\sigma_{1}}^{t}\left(\left|\frac{v_{1}(s)}{v_{1}(t)}\right|+\left|\frac{v_{2}(s)}{v_{2}(t)}\right|\right) a_{2}(s)\left|\gamma^{\varphi}(s)\right| d s
$$

which becomes again

$$
\left.\left.\left|\Delta_{2}(t)\right| \leqslant 2 \int_{\sigma_{1}}^{t} a_{2}(s) \cdot\left|\gamma^{\varphi}(s)\right| d s \quad \forall t \in\right] \sigma_{1}, \sigma_{2}\right]
$$

since $v_{i}=\check{\beta}_{\sigma_{i}}$ is nondecreasing, $i=1,2$.

Third case. $\left.t \in] \sigma_{2}, T\right], v_{1}(t)=\check{\beta}_{\sigma_{1}}(t)=\beta_{2}(t), v_{2}(t)=\check{\beta}_{\sigma_{2}}(t)=\beta_{2}(t)$. Thus

$$
\int_{\sigma_{2}}^{t} E(t, s) d s=0
$$

and from the first case

$$
\int_{0}^{\sigma_{1}} E(t, s) d s=0
$$

Thus

$$
\Delta_{2}(t)=\int_{0}^{\sigma_{1}} E(t, s) d s+\int_{\sigma_{1}}^{\sigma_{2}} E(t, s) d s+\int_{\sigma_{2}}^{t} E(t, s) d s \quad \forall t, \sigma_{2}<t \leqslant T
$$

becomes

$$
\Delta_{2}(t)=\int_{\sigma_{1}}^{\sigma_{2}} E(t, s) d s \quad \forall t, \sigma_{2}<t \leqslant T
$$

Thanks to (58.1), (62) gives:

$$
\left.\left.\left|\Delta_{2}(t)\right| \leqslant 2 . \int_{\sigma_{1}}^{\sigma_{2}} a_{2}(s) \cdot\left|\gamma^{\varphi}(s)\right| d s, \quad \forall t \in\right] \sigma_{2}, T\right] .
$$

By (60)

$$
\int_{0}^{T}\left|\Delta_{2}(t)\right|^{2} d t=\int_{\sigma_{1}}^{\sigma_{2}}\left|\Delta_{2}(t)\right|^{2} d t+\int_{\sigma_{2}}^{T}\left|\Delta_{2}(t)\right|^{2} d t .
$$

From (61) and using Cauchy-Schwarz inequality:

$$
\begin{aligned}
\int_{\sigma_{1}}^{\sigma_{2}}\left|\Delta_{2}(t)\right|^{2} d t & \leqslant 4 \int_{\sigma_{1}}^{\sigma_{2}}\left[\int_{\sigma_{1}}^{t} a_{2}(s)\left|\gamma^{\varphi}(s)\right| d s\right]^{2} d t \\
& \leqslant 4\left\|\gamma^{\varphi}\right\|_{\infty}^{2} \cdot\left(\sigma_{2}-\sigma_{1}\right)^{2} \cdot\left\|a_{2}\right\|_{L^{2}}^{2} .
\end{aligned}
$$


From (63) and after some similar computations, we obtain

$$
\int_{\sigma_{2}}^{T}\left|\Delta_{2}(t)\right|^{2} d t \leqslant 4\left\|\gamma^{\varphi}\right\|_{\infty}^{2} \cdot\left\|a_{2}\right\|_{\infty}^{2} \cdot\left(T-\sigma_{2}\right)\left(\sigma_{2}-\sigma_{1}\right)^{2} .
$$

Using (60), (63.1) and (63.2) we have:

$$
\int_{0}^{T}\left|\Delta_{2}(t)\right|^{2} d t \leqslant 4\left\|\gamma^{\varphi}\right\|_{\infty}^{2} \cdot\left(\sigma_{2}-\sigma_{1}\right)^{2}\left[\left\|a_{2}\right\|_{L^{2}}^{2}+T\left\|a_{2}\right\|_{\infty}^{2}\right] .
$$

By Young inequality we have for any $\theta>0$

$$
\left|a_{1}^{\prime}(t)-a_{2}^{\prime}(t)\right|^{2} \leqslant(1+\theta)\left|\Delta_{1}(t)\right|^{2}+\left(1+\frac{1}{\theta}\right)\left|\Delta_{2}(t)\right|^{2} .
$$

After integrating on $[0, T]$, using (63.3) and (59), it follows

$$
\begin{aligned}
\int_{0}^{T}\left|a_{1}^{\prime}(t)-a_{2}^{\prime}\right|^{2} d t \leqslant & (1+\theta)\left\|\gamma^{\varphi}\right\|_{\infty}^{2} \cdot \frac{T^{2}}{2}\left\|a_{1}-a_{2}\right\|_{L^{2}}^{2} \\
& +4\left(1+\frac{1}{\theta}\right) \cdot c \cdot\left\|\gamma^{\varphi}\right\|_{\infty}\left(\sigma_{2}-\sigma_{1}\right)^{2},
\end{aligned}
$$

where we have used the fact that $\left\|a_{2}\right\|_{L^{2}}^{2}+T$. $\left\|a_{2}\right\|_{\infty}^{2} \leqslant c$, constant independent of $\check{\beta}_{\sigma_{2}}$ by Proposition 2. Let us set

$$
2 \gamma_{0}=\frac{\lambda_{1}(1,1,0, T)-\left\|\gamma^{\varphi}\right\|_{\infty}^{2} \cdot T^{2} / 2}{\lambda_{1}(1,1,0, T)}
$$

By hypothesis $\left(\mathrm{H}_{2}^{\prime}\right), \gamma_{0}>0$ and

$$
\frac{\left\|\gamma^{\varphi}\right\|_{\infty}^{2} T^{2}}{2}<\lambda_{1}(1,1,0, T) .\left(1-\gamma_{0}\right)
$$

Let us choose $\theta>0$ small enough such that

$$
\delta=\left(1-\gamma_{0}\right) \lambda_{1}(1,1,0, T)-(1+\theta) \frac{\left\|\gamma^{\varphi}\right\|_{\infty}^{2} \cdot T^{2}}{2}>0 .
$$

From (63.4), using (63.5) and Appendix A, we obtain

$$
\gamma_{0}\left\|a_{1}^{\prime}-a_{2}^{\prime}\right\|_{L^{2}}^{2}+\delta\left\|a_{1}-a_{2}\right\|_{L^{2}}^{2} \leqslant c \cdot\left|\sigma_{1}-\sigma_{2}\right|^{2} .
$$

The second estimate is an easy consequence of (63.6). From Appendix B we can write

$$
a(\sigma, t)=a_{\sigma}(t)=\int_{T}^{t} a_{\sigma}^{\prime}(s) d s+1 \quad \forall \sigma, \forall t,
$$




$$
a(\sigma, t)-a\left(\sigma^{\prime}, t^{\prime}\right)=\int_{T}^{t}\left(a_{\sigma}^{\prime}(s)-a_{\sigma^{\prime}}^{\prime}(s)\right) d s+\int_{t^{\prime}}^{t} a_{\sigma^{\prime}}^{\prime}(s) d s .
$$

So

$$
\left|a(\sigma, t)-a\left(\sigma^{\prime}, t^{\prime}\right)\right| \leqslant c\left[\left|\sigma-\sigma^{\prime}\right|+\left|t-t^{\prime}\right|\right],
$$

using the first estimate and Appendix B that is $\left\|a_{\sigma^{\prime}}^{\prime}\right\|_{L^{\infty}(0, T)} \leqslant c$.

Now we are in position to prove the main result of this part.

THEOREM 3. - Assume hypothesis (15), (16), (H0), (H2), (H3) and (H4). Then the solution $u$ of

$$
\left\{\begin{array}{l}
-\Delta u+c . u=f \quad \text { in } \Omega \\
\left.u\right|_{\partial \Omega}=0
\end{array}\right.
$$

is positive.

Proof. - It is very similar to the one of Theorem 2. So we do not repeat it. To simplify the notations we denote by $\sigma$ the real number $\hat{\sigma}$ given by Proposition 7 . Let us recall that by Proposition 7 we have

$$
\ell^{\prime}(t):=\ell_{\sigma}^{\prime}(t) \leqslant 0, \quad \forall t \in[\sigma, \bar{t}] ; \quad \ell^{\prime}(t) \geqslant 0, \quad \forall t \in[0, \sigma] .
$$

To state our result, it sufficies to prove the inequality

$$
\left\{\begin{array}{l}
-\Delta \ell(\varphi)+c^{+} . \ell(\varphi) \leqslant f \quad \text { in } H^{-1}(\Omega) \\
\left.\ell(\varphi)\right|_{\partial \Omega}=0, \quad \ell(\varphi) \in H_{0}^{1}(\Omega)
\end{array}\right.
$$

where $\ell():.=\ell_{\sigma}($.$) is solution of$

$$
\left\{\begin{array}{l}
\left.-\ell^{\prime \prime}-\theta \cdot \ell^{\prime}+\gamma^{\varphi} \cdot \ell=\widetilde{f}_{\nabla, \varphi} \quad \text { in }\right] 0, \bar{t}[ \\
\ell^{\prime}(0)=0, \quad \ell(\bar{t})=0,
\end{array}\right.
$$

with $\left.\theta(t)=\check{\beta}_{\sigma}^{\prime}(t) / \check{\beta}_{\sigma}(t) \forall t \in\right] 0, \bar{t}[$.

For this we can assume that $\ell$ (.) is regular enough to define $\ell^{\prime \prime} \circ \varphi$ and $\ell^{\prime} \circ \varphi$ (cf. third step of the proof of Theorem 2), that is to say we do not write the regularization part of this proof. Recall that $\ell$ (.) has the following representation formula

$$
\ell(t)=a_{\sigma}(t) \cdot \int_{t}^{\bar{t}} \frac{d s}{\check{\beta}_{\sigma}(s) a_{\sigma}^{2}(s)} \int_{0}^{s} \check{\beta}_{\sigma} a_{\sigma} \widetilde{f}_{\nabla, \varphi}(\theta) d \theta,
$$

where $a_{\sigma}$ is solution of

$$
\left\{\begin{array}{l}
\left.-\frac{1}{\breve{\beta}_{\sigma}}\left(\check{\beta}_{\sigma} a_{\sigma}^{\prime}\right)^{\prime}+\gamma^{\varphi} \cdot a_{\sigma}=0 \quad \text { in }\right] 0, T[ \\
a_{\sigma}^{\prime}(0)=0, \quad a_{\sigma}(T)=1
\end{array}\right.
$$

Let us set 


$$
\begin{aligned}
& \Omega_{1}=\{x \in B(\bar{t}) \mid \varphi(x) \in] 0, \sigma[\}, \\
& \Omega_{2}=\{x \in B(\bar{t}) \mid \varphi(x) \in] \sigma, \bar{t}[\} .
\end{aligned}
$$

We have to examine two cases:

First case. For any $x \in \Omega_{1}$ set $t=\varphi(x)$ in (65). We have:

$$
-\ell^{\prime \prime}(\varphi)-\theta(\varphi) \ell^{\prime}(\varphi)+\gamma^{\varphi}(\varphi) \cdot \ell(\varphi)=\widetilde{f}_{\nabla, \varphi}(\varphi) \text { in } \Omega_{1}
$$

and

$$
\theta(\varphi(x))=\frac{\check{\beta}_{\sigma}^{\prime}(\varphi(x))}{\check{\beta}_{\sigma}(\varphi(x))}=\frac{\beta_{1}^{\prime}(\varphi(x))}{\beta_{1}(\varphi(x))}=\hat{\beta}_{\varphi}(\varphi(x)) \leqslant \hat{\beta}(x) \quad \text { in } \Omega_{1}
$$

since $0<\varphi(x)<\sigma \forall x \in \Omega_{1}$. But from Proposition 7, we have

$$
\ell^{\prime}(\varphi)=-\int_{0}^{\varphi} a_{\sigma} \beta_{\sigma} \widetilde{f}_{\nabla, \varphi} d s \cdot \frac{1}{a_{\sigma}^{2}(\varphi) \check{\beta}_{\sigma}(\varphi)} \geqslant 0 \quad \text { in } \Omega_{1}
$$

Thus, using (42.1), it follows

$$
-\theta(\varphi(x)) \ell^{\prime}(\varphi(x)) \geqslant-\hat{\beta}(x) \cdot \ell^{\prime}(\varphi(x))=-\frac{\Delta \varphi(x)}{|\nabla \varphi(x)|^{2}} \cdot \ell^{\prime}(\varphi(x)) \quad \text { in } \Omega_{1}
$$

or

$$
-\theta(\varphi) \ell^{\prime}(\varphi) \geqslant-\frac{\Delta \varphi}{|\nabla \varphi|^{2}} \ell^{\prime}(\varphi) \quad \text { in } \Omega_{1}
$$

In the same way we can prove that

$$
\gamma^{\varphi}(\varphi(x)) \ell(\varphi(x)) \geqslant \gamma(x) \cdot \ell(\varphi(x))=\frac{c^{+}(x)}{|\nabla \varphi(x)|^{2}} \ell(\varphi(x)) \text { in } B(\bar{t})
$$

since $\gamma^{\varphi}(\varphi) \leqslant \gamma$ in $B(\bar{t})$ and $\ell(\varphi)>0$ by Proposition 4. And also we have

$$
\widetilde{f}_{\nabla, \varphi}(\varphi(x)) \leqslant \frac{\tilde{f}(x)}{|\nabla \varphi(x)|^{2}} \leqslant \frac{f(x)}{|\nabla \varphi(x)|^{2}} \quad \text { in } B(\bar{t})
$$

by (21.1) and Definition 1. From $(67), 68)$ and (69), (66) becomes

$$
-\ell^{\prime \prime}(\varphi)|\nabla \varphi|^{2}-\Delta \varphi \ell^{\prime}(\varphi)+c^{+} . \ell(\varphi) \leqslant f \quad \text { in } \Omega_{1}
$$

or

$$
-\Delta \ell(\varphi)+c^{+} \ell(\varphi) \leqslant f \quad \text { in } \Omega_{1} .
$$

Second case. For any $x \in \Omega_{2}$, set $t=\varphi(x)$ in (65). Exactly in the same way as above we can prove that

$$
-\Delta \ell(\varphi)+c^{+} \ell(\varphi) \leqslant f \quad \text { in } \Omega_{2}
$$


since

$$
\theta(\varphi(x)) \geqslant \hat{\beta}(x) \text { in } \Omega_{2},
$$

and

$$
\ell^{\prime}(\varphi(x))=-\frac{\int_{0}^{\varphi} a_{\sigma} \beta_{\sigma} \widetilde{f}_{\nabla, \varphi} d s}{a_{\sigma}^{2}(\varphi) . \check{\beta}_{\sigma}(\varphi)} \leqslant 0 \quad \text { in } \Omega_{2},
$$

from Proposition 7. The remainder of the proof is now clear. In fact (70) and (71) "entail formally" inequality (64). To justify the above computations we proceed, as in Theorem 2, by regularization and use the extension lemma in Appendix C.

\section{General data in right hand side: role of the symmetrization}

Through the illustrative example of part (3) and the proof of Theorems 2 and 3, we have seen that the localization of the negative support $E_{f}$ plays a crucial role. It is natural to ask what happens if, for instance, the negative support $E_{f}$ is not easily localisable or is too close from the boundary of $\Omega$. For studying this question, we will use the notion of rearrangement in the sense of Hardy-Littlwood. This notion appears quite naturally in this situation. Unfortunately for the time being, the method used previously only works in ellipsoïdal and radial coordinates if we use the symmetrization techniques.

For convenience of the reader and precision of speech, we recall some elementary notions of rearrangement. Let $v$ be a bounded positive measure, absolutely continuous with respect to the Lebesgue measure on $[0, T]$. If $] 0, T$ [ is an open bounded subset of $\mathbb{R}$ and $v$ is a nonnegative function belonging to $L^{1}(] 0, T[)$, the distribution function of $v$, with respect to $v$, is the function $\mu_{v}$ defined by

$$
\mu_{v}(t)=v(\{s \in] 0, T[: v(s)>t\}), \quad t \in \mathbb{R}^{+} .
$$

For $s \in[0, T]$ we set

$$
v^{*}(s)=\sup \left\{t: \mu_{v}(t) \geqslant s\right\}
$$

$v^{*}$ is the decreasing rearrangement of $v$ with respect to the measure $v$. Let us set

$$
v_{*}(s)=v^{*}(T-s), \quad s \in[0, T] .
$$

$v_{*}$ is the increasing rearrangement of $v$ with respect to the measure $v$. Let us now recall some results about rearrangements that we need in the sequel (cf. $[2,8])$.

Proposition 8. - Let $v \in L^{1}(] 0,1[, v)$. For any v-measurable subset $E$ of $[0, T]$ we have:

(i) $\left(\left.v\right|_{E}\right)_{*}(s) \geqslant\left(\left.v_{*}\right|_{[0, v(E)]}\right)(s) \forall s \in[0, v(E)]$, where $\left.v\right|_{E}$ stands for the restriction of $v$ to $E$,

(ii) $\left(\left.v\right|_{E}\right)^{*}(s) \leqslant\left(\left.v^{*}\right|_{[0, v(E)]}\right)(s) \forall s \in[0, v(E)]$.

The proof is left to the reader (cf. for instance $[8,2]$ ). 
Consider the general setting of the problem in Section 6. We do not assume the localization hypothesis of the negative support of $E_{f}$. To simplify the notations, let us set

$$
\begin{aligned}
& g(t)=\widetilde{f}_{\nabla, \varphi}(t)=\left(\frac{\tilde{f}}{|\nabla \varphi|^{2}}\right)_{\varphi}(t), \quad \forall t \in[0, T] ; \\
& g(t)=g^{+}(t)-g^{-}(t), \quad \forall t \in[0, T] .
\end{aligned}
$$

To simplify the exposition, let us assume that $g$ belongs to $L^{\infty}([0, T])$ and let us consider the measure $v$ defined as follows: for any measurable subset $E$ of $[0, T]$

$$
v(E)=\int_{E} a \beta(s) d s .
$$

Proposition 9. - We have

$$
\int_{0}^{t} g d v=\int_{0}^{t} a \beta g d s \geqslant \int_{0}^{\gamma(t)}\left[\left(g^{+}\right)_{*}(s)-\left(g^{-}\right)^{*}(s)\right] d s
$$

where $\gamma(t)=v([0, t])=\int_{0}^{t} a(\theta) \beta(\theta) d \theta$ and where the rearrangements of $g^{+}$and $g^{-}$ are taken with respect to $\nu$.

The proof is a straightforward consequence of the equimeasurability property of the rearrangements and of Proposition 8.

Proposition 10. - Let us consider the function

$$
\omega(t)=\int_{t}^{T} \frac{1}{a^{2}(s) \beta(s)} \int_{0}^{\gamma(s)}\left[\left(g^{+}\right)_{*}-\left(g^{-}\right)^{*}\right](\sigma) d \sigma d s .
$$

It is well defined on $[0, T]$.

Proof. - It suffices to prove that $\omega$ is well defined at $t=0$. We have

$$
0 \leqslant \int_{0}^{T} \frac{1}{a^{2} \beta} \int_{0}^{\gamma(s)}\left(g^{+}\right)_{*}(\sigma) d \sigma d s \leqslant \int_{0}^{T} \frac{1}{a^{2} \beta}\left(g^{+}\right)_{*}(\gamma(s)) \cdot \gamma(s) d s
$$

since $\left(g^{+}\right)_{*}$ is nondecreasing. Thus

$$
0 \leqslant \int_{0}^{T} \frac{1}{a^{2} \beta} \int_{0}^{\gamma(s)}\left(g^{+}\right)_{*}(\sigma) d \sigma d s \leqslant c \cdot \int_{0}^{T} \frac{\left(g^{+}\right)_{*}(\gamma(s))}{a^{2}(s)} d s
$$

since

$$
\gamma(s)=\int_{0}^{s} a(\sigma) \beta(\sigma) d \sigma \leqslant \beta(s) \int_{0}^{s} a(\sigma) d \sigma \leqslant \beta(s) . \int_{0}^{T} a(\sigma) d \sigma \leqslant c . \beta(s)
$$


by using the fact that $\beta$ is nondecreasing. In the same way we can prove that

$$
0 \leqslant \int_{0}^{T} \frac{1}{a^{2} \beta} \int_{0}^{\gamma(s)}\left(g^{-}\right)^{*}(\sigma) d \sigma d s \leqslant c . \int_{0}^{T} \frac{\left(g^{-}\right)^{*}(0)}{a^{2}(s)} d s .
$$

Consequently (72) and (73) imply the claimed result.

Consider the function $h(s)=\left(g^{+}\right)_{*}(s)-\left(g^{-}\right)^{*}(s)$. Since $g^{+} \not \equiv 0$ and $g^{-} \not \equiv 0$, there exists $t \in] 0, v([0, T])]$ such that

$$
\begin{array}{ll}
h(s) \leqslant 0 & \text { a.e. } s \in[0, t[ \\
h(s) \geqslant 0 & \text { a.e. } s \in] t, v([0, T])] .
\end{array}
$$

Set $t_{0}=\inf \left[t \in[0, \gamma(T) / h(t) \geqslant 0]\right.$. It is clear that $t_{0}>0$ since $g^{-} \not \equiv 0$. So we have

$$
\begin{cases}h(s) \leqslant 0 & \text { in }\left[0, t_{0}\right] \\ h(s) \geqslant 0 & \text { in } \left.] t_{0}, \gamma(T)\right]\end{cases}
$$

Let us point out that $\left[0, t_{0}\right]$ contains the negative support $E_{h}$ of $h$; and thus $E_{h}$ is localized. Then a natural question arises: what is the hypothesis (H1) becoming?

Answer: (H1) becomes ( $\left.\mathrm{H}^{\prime} 1\right)$ : there exists $\left.\left.\bar{t} \in\right] t_{0}, T\right]$ such that:

$$
\int_{0}^{\bar{t}} \frac{1}{a^{2} \beta(s)} \int_{0}^{\gamma(s)}\left[\left(g^{+}\right)_{*}-\left(g^{-}\right)^{*}\right](\sigma) d \sigma d s>0 .
$$

PROPOSITION 11. - Under $\left(\mathrm{H}^{\prime} 1\right)$ the function

$$
\hat{v}(t)=\int_{t}^{\bar{t}} \frac{1}{a^{2}(s) \beta(s)} \int_{0}^{\gamma(s)}\left[\left(g^{+}\right)_{*}-\left(g^{-}\right)^{*}\right](\sigma) d \sigma d s
$$

is positive in $] 0, \bar{t}[$.

Proof. - Consider $G(t)=\int_{0}^{t}\left[\left(g^{+}\right)_{*}-\left(g^{-}\right)^{*}\right](\sigma) d \sigma$, defined for any $t$ belonging to the range $[0, \gamma(\bar{t})]$ of $\gamma$. Since $E_{h}$ is localized, from $\left(\mathrm{H}^{\prime} 1\right)$ there exists a unique $\tilde{t}$ such that:

$$
\begin{cases}G(\tilde{t})=0, & \\ G(t)<0 & \forall t \in[0, \tilde{t}[ \\ G(t)>0 & \forall t \in] \tilde{t}, \gamma(\bar{t})] .\end{cases}
$$

And since $\gamma$ is continuous and increasing, there exists a unique $\hat{s} \in] t_{0}, \bar{t}[$ such that $\gamma(\hat{s})=\tilde{t}$, that is

$$
\begin{aligned}
& G \circ \gamma(\hat{s})=0, \\
& G \circ \gamma(s)<0 \quad \forall t \in[0, \hat{s}[, \\
& G \circ \gamma(s)>0 \quad \forall t \in] \hat{s}, t] .
\end{aligned}
$$


But $\left.\hat{v}^{\prime}(t)=-G \circ \gamma(t) /\left(a^{2}(t) \beta(t)\right) \forall t \in\right] 0, \bar{t}[$, which means that

$$
\begin{cases}\hat{v}^{\prime}(\hat{s})=0, & \\ \hat{v}^{\prime}(s)>0 & \text { in }[0, \hat{s}[ \\ \hat{v}^{\prime}(s)<0 & \text { in }] \hat{s}, \bar{t}[\end{cases}
$$

Since from $\left(\mathrm{H}^{\prime} 1\right) \hat{v}(0)>0$ and since $\hat{v}(\bar{t})=0$, the result follows.

THEOREM 4. - Let us assume $\left(\mathrm{H}^{\prime} 1\right)$. Then the solution u of Eq. (1) is positive in $\Omega$.

Proof. - Consider the following function

$$
v(t)=\int_{t}^{\bar{t}} \frac{1}{a^{2}(s) \beta(s)}\left(\int_{0}^{s} g a \beta d \sigma\right) d s, \quad \forall t \in[0, \bar{t}] .
$$

From Proposition 9 we have:

$$
v(t) \geqslant \hat{v}(t) \quad \forall t \in[0, \bar{t}]
$$

since $a^{2}(t) \beta(t)>0 \forall t>0$. Thus the solution $\ell(t)=a(t) \cdot v(t)$ of (22) is positive in $] 0, \bar{t}$. Now, proceeding as in Theorem 2 of the Section 4, we can construct, from $\ell$ (.), a positive subsolution of (1). And the result follows from the maximum principle.

\section{Applications}

In this section, our main goal is to give some direct applications of our result.

\subsection{Averaging positivity}

Let us consider the following problem

$$
\left\{\begin{array}{l}
-\Delta u-c(x)|u|=f \quad \text { in } \Omega=B(0, R), \\
\left.u\right|_{\partial \Omega}=0
\end{array}\right.
$$

where $c(x)$ is a regular function such that:

$$
0<\alpha \leqslant c(x)<\lambda_{1}(\Delta, B), \quad \forall x \in \Omega
$$

where $\alpha$ is a positive constant and $\lambda_{1}(\Delta, B)$ the first eigenvalue of $\Delta$ in $B$ with Dirichlet condition. We assume that problem (75) has a solution $u$. We denote by $\gamma(|x|)$ the following function

$$
\gamma(r)=\inf \left[\frac{\widetilde{c .|v|}(r)}{|\widetilde{v}|(r)} \mid v \text { regular }\right]
$$


where, for any regular function $h$, we set

$$
\widetilde{h}(x)=\int_{S O(n)} h(A x) d \mu(A)
$$

with $\mu$ is a left- (or right)-Haar measure on $S O(n)$, the rotational group in $\mathbb{R}^{n}, A \in S O(n)$. We have $\tilde{h}(x)=\tilde{h}(|x|)-($ cf. $[18,19])$. It is easy to see that

$$
\lambda_{1}(\Delta, B)>\gamma(|x|) \geqslant \alpha, \quad \forall x \in \Omega .
$$

Then we consider the following radial problem:

$$
\left\{\begin{array}{l}
-\Delta v=\tilde{f}(|x|) \quad \text { in } B(0, R) \\
\left.v\right|_{\partial B}=0
\end{array}\right.
$$

where $\widetilde{f}(r)$ is defined previously.

Similarly to (7), we assume that:

$$
\begin{gathered}
\int_{0}^{R}\left[\int_{0}^{\sigma} \tilde{f}(r) r^{n-1} d r\right] \frac{d \sigma}{\sigma^{n-1}}>0, \\
\{r \in[0, R] \mid \widetilde{f}(r)<0\}=\left[0, r_{1}\right], \quad r_{1}<R .
\end{gathered}
$$

From our Theorem 1, we have

$$
v(x)=v(|x|)>0, \quad \forall x \in B(0, R) .
$$

We have

$$
\left\{\begin{array}{l}
-\Delta v-\gamma(|x|) v \leqslant \tilde{f}(|x|) \quad \text { in } B(0, R), \\
\left.v\right|_{\partial B}=0
\end{array}\right.
$$

since $\Delta \tilde{u}=\widetilde{\Delta u}-($ see $[19])$. It is well known that, for any $\omega \in H^{1}(B)$, we can define $\widetilde{\omega}$. Thus $\widetilde{u}$ is well defined and we have

$$
\left\{\begin{array}{l}
-\Delta \widetilde{u}-\widetilde{c|u|}=\widetilde{f} \quad \text { in } B(0, R), \\
\left.\widetilde{u}\right|_{\partial B}=0 .
\end{array}\right.
$$

Let us state an estimate from above of the left hand side of (78).

$$
-\Delta \widetilde{u}-\gamma(|x|) \widetilde{u} \geqslant-\Delta \widetilde{u}-\gamma(|x|)|\widetilde{u}| \geqslant \widetilde{f}=-\Delta \widetilde{u}-\widetilde{c|u|}
$$

since $\widetilde{c|u|} \geqslant \gamma(|x|) . \widetilde{|u|}$; i.e., $\widetilde{u}$ solves the following inequality

$$
\left\{\begin{array}{l}
-\Delta \widetilde{u}-\gamma(|x|) \widetilde{u} \geqslant \widetilde{f} \geqslant-\Delta v-\gamma(|x|) . v \quad \text { in } B(0, R), \\
\left.\widetilde{u}\right|_{\partial B}=0=\left.v\right|_{\partial B}
\end{array}\right.
$$


after using (77.1). A straightforward application of the maximum principle gives the result:

$$
\left.\frac{1}{\sigma_{r}} \cdot \int_{|x|=r} u(x) d \sigma>0, \quad \forall r \in\right] 0, R[.
$$

Consequently we have proved that if $f$ satisfies (76) and (77), then the solution $u$ of (75) is such that $\widetilde{u}(|x|)>0$ in $] 0, R[$.

\subsection{Biharmonic operator}

To simplify, we take $\Omega=B(0, R)$ and we consider

$$
\left\{\begin{array}{l}
\Delta^{2} u=f \quad \text { in } B(0, R) \\
\left.u\right|_{\partial B}=\left.\Delta u\right|_{\partial B}=0
\end{array}\right.
$$

We denote, for any $x \in B(0, R)$

$$
k(|x|)=\sup \{h(|x|) \mid h \text { radial function s.t. } h(|\cdot|) \leqslant f(\cdot) \text { on } B(O, R)\} ;
$$

and we consider the Green function $G$ of $-\Delta$ in $B(0, R)$. We assume that $f$ satisfies assumptions (80) and (82) given here after:

$$
\int_{B} k(|y|)\left[\int_{B} G(0, z) G(z, y) d z\right] d y>0,
$$

there exists $r_{0} \in[0, R[$ such that the function

$$
K(r)=\int_{0}^{r} k(s) s^{N-1} d s
$$

verifies:

$$
\begin{cases}K(r)<0, & \text { on }\left[0, r_{0}[\right. \\ K(r) \geqslant 0, & \text { on }\left[r_{0}, R[\right.\end{cases}
$$

Our goal is to prove that, under assumptions (80) and (82), the solution $u$ of the biharmonic problem (79) is positive. Setting

$$
\omega=-\Delta u,
$$

we have:

$$
\left\{\begin{array}{l}
-\Delta u=\omega, \\
\left.u\right|_{\partial B}=0,
\end{array}\right.
$$

and

$$
\left\{\begin{array}{l}
-\Delta \omega=f \\
\left.\omega\right|_{\partial B}=0 .
\end{array}\right.
$$


Let us now consider the solution $v$ of:

$$
\left\{\begin{array}{l}
\Delta^{2} v=k \\
\left.v\right|_{\partial B}=\left.\Delta v\right|_{\partial B}=0
\end{array}\right.
$$

and let us set

$$
m=-\Delta v
$$

We have:

$$
\left\{\begin{array}{l}
-\Delta m=k \\
\left.m\right|_{\partial B}=0
\end{array}\right.
$$

and

$$
\left\{\begin{array}{l}
-\Delta v=m \\
\left.v\right|_{\partial B}=0
\end{array}\right.
$$

This function $m$ is radial and satisfies:

$$
\begin{aligned}
& m(|x|) \leqslant \omega(x) \quad \text { in } B(0, R), \\
& m(|x|)=\int_{|x|}^{R} \frac{1}{\sigma^{N-1}}\left[\int_{0}^{\sigma} k(r) r^{N-1} d r\right] d \sigma=\int_{|x|}^{R} \frac{1}{\sigma^{N-1}} K(\sigma) d \sigma .
\end{aligned}
$$

From (82), it is clear that:

$$
\left\{\begin{array}{l}
m(|x|)>0 \quad \forall x,|x|>r_{0}, \\
\{x \in B(0, R) \mid m(|x|)<0\}=\left[0, r_{1}[\right.
\end{array}\right.
$$

for some $r_{1}$ in $\left[0, r_{0}\right]$. In Eqs. (87), (88) and hypothesis (80) play, respectively, the roles of localization hypothesis (7.1) and (7.2) if we want to apply Theorem 1. Thus $v$ is positive, and so is $u$ since we have

$$
u(x) \geqslant v(|x|) \quad \forall x \in B(0, R) .
$$

Remarks. -

(i) it is possible to assume hypothesis ensuring positivity of $m$ by application of Theorem 1 . But in this case, the positivity of $u$ is immediate since $v$ is obviously positive.

(ii) assumptions (80) and (82) are only simple sufficient conditions ensuring positivity of $u$. Here, our aim is not to give general conditions ensuring positivity of $u$ but to explain clearly how to use our result in some applications. It is possible to improve notably conditions (80) and (82).

(iii) using (81) and (87.1) it is possible to explain the condition (80) more explicitly with respect to $k(\cdot)$.

\subsection{A negative result}

The following example shows, if it is necessary, that hypothesis (7) and (H1) do not seems related to the method. Let us consider

$$
\left\{\begin{array}{l}
-\Delta u=f(|x|) \quad \text { in } \Omega, \\
\left.u\right|_{\Gamma}=0, \quad \Gamma=\partial \Omega
\end{array}\right.
$$


Let $B(0, R)$ be the smallest open ball containing $\Omega$ and $B(O, r)$ the largest ball enclosed in $\bar{\Omega}$. We assume that $f(|x|)$ is nonnegative in $\Omega \backslash B(O, r)$. Let us denote by $\widetilde{f}(|x|)$ the natural extension of $f$ to $B(0, R)$ and let us assume that we have

$$
\begin{gathered}
\int_{0}^{R} \frac{d \sigma}{\sigma^{n-1}}\left[\int_{0}^{\sigma} \widetilde{f}(r) r^{n-1} d r\right]<0 \\
\left.\frac{\partial u}{\partial \eta}\right|_{\partial \Omega}<0 .
\end{gathered}
$$

Then $u$ is nonpositive in a neighbourhood of 0 . Indeed let us consider the solution $v$ of

$$
\left\{\begin{array}{l}
-\Delta v=\widetilde{f}(|x|) \quad \text { in } B(0, R) \\
\left.v\right|_{\partial B}=0
\end{array}\right.
$$

Set

$$
\widetilde{u}(x)= \begin{cases}u(x) & \text { if } x \in \Omega, \\ 0 & \text { if } x \in B(0, R) \backslash \bar{\Omega} .\end{cases}
$$

From (91) and the extension lemma (cf. Appendix C), we deduce that $\widetilde{u}$ is a subsolution of (92). Thus from the maximum principle $u$ is nonpositive in a neighbourhood of 0 since $v(0)<0$ by (90). Finally let us point out that we can state a more general negative result [16].

\subsection{Localization of critical points}

Let $\Omega$ be an open subset of $\mathbb{R}^{n}, \Omega=\Omega_{1} \backslash \bar{\Omega}_{2}, \bar{\Omega}_{2} \subset \Omega_{1}$, where $\Omega_{i}$ is star-shaped with respect to 0 . In this geometric situation there exist some hypothesis satisfied by $f$ and ensuring that the level sets of the solution $u$ of

$$
\left\{\begin{array}{l}
-\Delta u=f \quad \text { in } \Omega \\
\left.u\right|_{\partial \Omega}=0,
\end{array}\right.
$$

are star-shaped $[3,14,4]$ (for instance). Let us point out that the method used in the previous references does not work if $\Omega$ is simply connected. Here our goal is to give some results about star-shapedness of $u$ in the case $\Omega$ is star-shaped with respect to some ball $B(0, r)$ (or with respect to 0 ). Without loss of generality of our method, we can assume that $f(x)=f(|x|)$ in $\mathbb{R}^{n}$ and is regular enough. Suppose that $u$ has a local maximum at 0 . Assume that $f$ is nonnegative and satisfies the following localization hypothesis: considering the subset $E$ of $\Omega$,

$$
E=\left\{x \in \Omega|-2 f(|x|)-| x \mid \cdot f^{\prime}(|x|)<0\right\},
$$

there exists $r_{0}>0$ such that

$$
E \subseteq B\left(0, r_{0}\right) \subset \Omega .
$$


For some $\rho>0$ such that $B\left(0, r_{0}\right) \subset B(0, \rho) \subseteq \Omega$, let us consider $v_{\rho}$ the solution of

$$
\left\{\begin{array}{l}
-\Delta v_{\rho}=g(|x|) \quad \text { in } B(0, \rho) \\
\left.v_{\rho}\right|_{\partial B(0, \rho)}=0
\end{array}\right.
$$

with $g(|x|)=-2 f(|x|)-|x| \cdot f^{\prime}(|x|)$. Set

$$
\widetilde{v}_{\rho}(x)= \begin{cases}v_{\rho}(x) & \text { if } x \in B(0, \rho), \\ 0 & \text { if } x \notin B(0, \rho) .\end{cases}
$$

Now let us remark that $\omega(x)=x \cdot \nabla u(x)$ is solution of

$$
\left\{\begin{array}{l}
-\Delta \omega=g(|x|) \quad \text { in } \Omega \\
\left.\omega\right|_{\partial \Omega}>0
\end{array}\right.
$$

where the positivity of the boundary condition follows from Hopf maximum principle.

THEOREM 5 (Necessary conditions).-Consider $\omega$ and $\widetilde{v}_{\rho}$ and assume that $u$ is regular in a neighbourhood of 0 .

(1) If $\widetilde{v}_{\rho}$ is a subsolution of (96) then we have

$$
\int_{0}^{\rho}\left[\int_{0}^{\sigma} g(s) s^{n-1} d s\right] \frac{d \sigma}{\sigma^{n-1}} \leqslant 0 .
$$

(2) If $\widetilde{v}_{\rho}$ is a positive subsolution of (96), then

$$
\int_{0}^{\rho}\left[\int_{0}^{\sigma} g(s) s^{n-1} d s\right] \frac{d \sigma}{\sigma^{n-1}}=0 .
$$

(3) Suppose that $g$ has the localization hypothesis (94) and $\widetilde{v}_{\rho}$ is a positive subsolution of (96), then

$$
\rho=\sup \{r>0 \mid B(0, r) \subseteq \Omega\} .
$$

Proof. $-v_{\rho}$ has the following representation formula

$$
v_{\rho}(x)=v_{\rho}(|x|)=\int_{|x|}^{\rho}\left[\int_{0}^{\sigma} g(s) s^{n-1} d s\right] \frac{d \sigma}{\sigma^{n-1}}
$$

and $v_{\rho}(0) \leqslant \omega(0)=0$ since $u$ is regular at 0 . If $\widetilde{v}_{\rho}(x) \geqslant 0 \forall x \in \Omega$, it follows that $v_{\rho}(0)=0$. To prove the third part of our result, we argue by contradiction, i.e., assume that there exists $\rho, 0<r_{0}<\rho<R$ such that $\widetilde{v}_{\rho}$ is a positive subsolution of (96) and where

$$
R=\sup \left\{r>r_{0} \mid B(0, r) \subseteq \Omega\right\} .
$$

From the localization hypothesis (94) we have 


$$
\begin{aligned}
& 0<v_{\rho}(x)<v_{R}(x) \quad \forall x \in B(0, \rho), x \neq 0, \\
& 0 \leqslant \widetilde{v}_{\rho}(x) \leqslant \widetilde{v}_{R}(x) \quad \forall x \in \Omega,
\end{aligned}
$$

by using maximum principle and extension lemma in Appendix $\mathrm{C}$, where $\widetilde{v}_{R}$ is defined similarly as $\widetilde{v}_{\rho}$. We have

$$
0=\widetilde{v}_{\rho}(0)=v_{\rho}(0)<v_{R}(0)=\widetilde{v}_{R}(0),
$$

by using the second part of the result. Thus

$$
v_{R}>0 \quad \forall x \in B(0, R)
$$

and therefore $\widetilde{v}_{R}($.$) is a positive subsolution of (96) from Theorem 1$. So we have

$$
0<v_{R}(0)=\lim _{x \rightarrow 0} v_{R}(x) \leqslant \lim _{x \rightarrow 0} \omega(x)=\omega(0)
$$

which contradicts the fact that $\omega(0)=0$ since $u$ is regular at 0 .

Now, from the above result it is necessary to assume:

$$
\begin{aligned}
& \int_{0}^{R}\left[\int_{0}^{\sigma} g(s) s^{n-1} d s\right] \frac{d \sigma}{\sigma^{n-1}}=0, \\
& R=\sup \left\{r \geqslant r_{0} \mid B(0, r) \subseteq \Omega\right\} .
\end{aligned}
$$

From (94) and (97) it follows that

$$
\int_{0}^{R} g(s) s^{n-1} d s>0
$$

COROLlary. - Assume that $u$ is regular and 0 is a point where $u$ has a local maximum. Suppose hypothesis (94), (97), (98) and (99). Then 0 is the only critical point of $u$. In addition the level sets

$$
F(t)=\{x \in \Omega \mid u(x)>t\}
$$

of $u$ are star-shaped with respect to 0 .

Proof. - It sufficies to remark that (94) and (97) imply that

$$
v_{R}(x)=v_{R}(|x|)=\int_{|x|}^{R}\left[\int_{0}^{\sigma} g(s) s^{n-1} d s\right] \frac{d \sigma}{\sigma^{n-1}}>0, \quad \forall x \in B(0, R), x \neq 0 .
$$

From Theorem 1 it follows that

$$
\omega(x)=x . \nabla u(x)>0 \quad \forall x \in \Omega, x \neq 0 ;
$$


that is to say $|\nabla u(x)| \neq 0 \forall x \neq 0$. We deduce the last part of the result from the implicit function theorem.

Remark. - Let us point out that hypothesis (97) is somewhat strong. In a forthcoming work [16] we propose to relax it by considering the function $x . \nabla u(x) /|x|^{2}, x \neq 0$, which is more pertinent than the function $x . \nabla u(x)$ used in this paper. But the study of the equation satisfied by $x . \nabla u(x) /|x|^{2}$ requires a preliminary investigation.

\subsection{Positive solutions of semi-linear problems}

We are concerned with the positivity of the solution $u$ of

$$
\left\{\begin{array}{l}
-\Delta u=f(x, u) \quad \text { in } \Omega=B(0, R), \\
\left.u\right|_{\partial B}=0,
\end{array}\right.
$$

for instance, for some suitable $f: \Omega \times \mathbb{R} \rightarrow \mathbb{R}$, ensuring the existence of $u$. Let us set

$$
g(r)=\inf [f(x, u(x))|x \in B(0, R),| x \mid=r]
$$

and suppose that $g=g^{+}-g^{-}$belongs to $L^{2}(\Omega)$. Let us consider the increasing rearragement $\left(g^{+}\right)_{*}$ of $g^{+}$and the decreasing rearrangement $\left(g^{-}\right)^{*}$ of $g^{-}$, with respect to the measure $d v=s^{n-1} d s$. Assume that

$$
\int_{0}^{R} \frac{d \sigma}{\sigma^{n-1}} \cdot \int_{0}^{\gamma(\sigma)}\left[\left(g^{+}\right)_{*}-\left(g^{-}\right)^{*}\right](s) d s>0
$$

where $\gamma(\sigma)=\int_{0}^{\sigma} s^{n-1} d s$. Proceeding in the same way as in Example 4, we can see that the function

$$
\hat{v}(|x|)=\int_{|x|}^{R} \frac{d \sigma}{\sigma^{n-1}} \int_{0}^{\gamma(\sigma)}\left[\left(g^{+}\right)_{*}-\left(g^{-}\right)^{*}\right](s) d s
$$

is positive. Taking $d v=s^{n-1} d s$ in Proposition 9, Section 5, we obtain

$$
v(|x|) \geqslant \hat{v}(|x|) \quad \forall x \in B(0, R)
$$

where

$$
v(|x|)=\int_{|x|}^{R} \frac{d \sigma}{\sigma^{n-1}} \int_{0}^{\sigma} g(s) s^{n-1} d s
$$

is the solution of:

$$
\left\{\begin{array}{l}
-\Delta v=g(|x|) \quad \text { in } B(0, R), \\
\left.v\right|_{\partial B}=0 .
\end{array}\right.
$$

From (101), $v$ is a subsolution of (100). Thus

$$
u(x) \geqslant v(|x|)>0 \quad \forall x \in B(0, R) .
$$




\subsection{Parabolic equation}

Here we are concerned with the positivity of the solution of linear parabolic equation. We need to start with some preliminary remarks. let us consider the following equation

$$
\left\{\begin{array}{l}
\left.\frac{\partial u}{\partial t}-\frac{\partial^{2} u}{\partial x^{2}}=f(x) \quad \text { in }\right]-1,1[\times] 0, T[ \\
u(-1)=u(1)=0, \quad u(x, 0)=u_{0}(x) .
\end{array}\right.
$$

Let us assume that $f$ is nonnegative and belongs to $C^{\infty}([-1,1])$. Suppose that $u_{0}$ belongs to $C^{\infty}([-1,1])$ and satisfies the following: there exists $\left.[a, b] \subset\right]-1,1[$ such that $u_{0}(x)<0 \forall x \in[a, b]$. Then from regularity result (cf. [1] Theorem X.10) it follows that there exists $t_{0}, 0<t_{0}<T$ such that $u(x, t)<0, \forall(x, t) \in[a, b] \times\left[0, t_{0}\right]$. Even if $f$ is nonnegative, the previous result shows that it is necessary to impose some assumption on $u_{0}$, to obtain the positivity of $u$. Now let us consider

$$
\left\{\begin{array}{l}
\left.\frac{\partial u}{\partial t}-\Delta u=f(x, t) \quad \text { in } B(0, R) \times\right] 0, T[, \\
\left.\left.u\right|_{\Sigma}=0, \quad \Sigma=\partial \Omega \times\right] 0, T\left[, \quad u(x, 0)=u_{0} .\right.
\end{array}\right.
$$

Let us assume that there exists $g(|x|)$ such that:

$$
\begin{aligned}
& f(x, t) \geqslant g(|x|) \quad \text { a.e. }(x, t) \in B(0, R) \times] 0, T[, \\
& \int_{0}^{R} \frac{d s}{s^{n-1}} \int_{0}^{s} \sigma^{n-1} g(s) d s \geqslant 0, \\
& E_{g} \subseteq B\left(0, r_{0}\right) \subset B(0, R) .
\end{aligned}
$$

Suppose

$$
u_{0}(x) \geqslant v(x)=v(|x|) \quad \text { a.e. } x \in B(0, R),
$$

where $v$ is the solution of

$$
\left\{\begin{array}{l}
-\Delta v=g(|x|) \quad \text { in } B(0, R) \\
\left.v\right|_{\partial B}=0
\end{array}\right.
$$

From our previous results we have $v(x) \geqslant 0$. Thus $\omega=u-v$ satisfies

$$
\left\{\begin{array}{l}
\left.\frac{\partial \omega}{\partial t}-\Delta \omega=f(x, t)-g(|x|) \geqslant 0 \quad \text { in } B(0, R) \times\right] 0, T[ \\
\left.\omega\right|_{\partial \Sigma}=0, \quad \omega(x, 0)=u_{0}(x)-v(x) \geqslant 0 \quad \text { in } B(0, R) .
\end{array}\right.
$$

Finally, the positivity of $u$ follows from maximum principle of parabolic equation (cf. $[5,9])$.

Remark. - The condition (103) cannot be improved. In fact, let us consider a function $v \in C^{2}([-1,+1]) \cap H_{0}^{1}(]-1,+1[)$ such that: 


$$
\begin{aligned}
& v(0)=v(-1)=v(1)=0, \quad v^{\prime \prime}(-\theta)=v^{\prime \prime}(\theta)=0, \quad \text { for some } \theta>0, \\
& \left.v^{\prime \prime}(x)<0 \quad \forall x \in[-1,-\theta[\cup] \theta, 1], \quad v^{\prime \prime}(x)>0 \quad \forall x \in\right]-\theta, \theta[.
\end{aligned}
$$

Let us set $f(x, t):=-v^{\prime \prime}(x)$ and let $u_{0}$ be such that

$$
\left\{\begin{array}{l}
\left.-u_{0}^{\prime \prime}(x)<f(x) \quad \text { in }\right]-1,+1[ \\
u_{0}(-1)=u_{0}(1)=0, \quad u_{0} \in C^{2}([-1,+1])
\end{array}\right.
$$

It is clear that there exists $\eta>0$ such that $\left.u_{0}(x)<0 \forall x \in\right]-\eta, \eta[$. And thus the solution $u$ of (102) is negative in a neighbourhood of 0 , for $t>0$ small enough.

\subsection{Extension to general elliptic operator}

It is possible to extend our result (Theorems 1,2) to operators of the form

$$
A=\sum_{i j} a_{i j}(x) \frac{\partial^{2}}{\partial x_{i} \partial x_{j}}+\sum_{i} b_{i}(x) \frac{\partial}{\partial x_{i}}+c(x) .
$$

Formally our method works. The difficulties are technical.

\section{Appendix A. First eigenvalue of a degenerate operator}

Let $\beta_{1}, \beta_{2}, \beta$ be three positive continuous functions defined on $[0, T]$, such that

$$
\left\{\begin{array}{l}
0<\beta_{2}(t) \leqslant \beta(t) \leqslant \beta_{1}(t) \quad \forall t>0, \\
c . \beta_{2}(t) \geqslant \beta(t) \quad \forall t>0, \quad c=\text { constant }>0, \\
\beta(0)=0 .
\end{array}\right.
$$

We assume that $\beta$ is nondecreasing. We have the following result

PROPOSITION A.1.-

$$
\lambda_{1}(\beta, \beta, 0, T)=\inf \left[\frac{\int_{0}^{T} \beta\left(v^{\prime}\right)^{2} d x}{\int_{0}^{T} \beta v^{2} d x} \mid v \in V\right] \geqslant \frac{1}{T^{2}}>0
$$

where

$$
V=\{v \text { defined on }] 0, T\left[\mid \sqrt{\beta} v \text { and } \sqrt{\beta} v^{\prime} \in L^{2}(] 0, T[), v(T)=0\right\} .
$$

In addition the map $T \rightarrow \lambda_{1}(\beta, \beta, 0, T)$ is nonincreasing and $\lambda_{1}(\beta, \beta, 0, T)$ tends to $+\infty$ as $T$ goes to $0^{+}$.

Proof. - Let us remark that for any $v$ belonging to $V, v \in H^{1}(] \varepsilon, T[) \forall \varepsilon>0$. Thus we have

$$
\forall x \in] 0, T\left[, \forall u \in V, \quad \sqrt{\beta(x)} u(x)=-\int_{x}^{T} \sqrt{\beta(x)} \cdot u^{\prime}(s) d s\right.
$$




$$
\begin{aligned}
\sqrt{\beta(x)}|u(x)| & \leqslant \int_{x}^{T} \sqrt{\beta(x)}\left|u^{\prime}(s)\right| d s \leqslant \int_{x}^{T} \sqrt{\beta(s)}\left|u^{\prime}(s)\right| d s \\
& \leqslant \int_{0}^{T} \sqrt{\beta(s)}\left|u^{\prime}(s)\right| d s,
\end{aligned}
$$

since $\beta($.$) is nondecreasing. Using Cauchy-Schwarz inequality we obtain:$

$$
\forall x>0, \quad \beta(x)|u(x)|^{2} \leqslant T . \int_{0}^{T} \beta(s)\left|u^{\prime}(s)\right|^{2} d s,
$$

and by integraton it follows

$$
\frac{1}{T^{2}} \leqslant \frac{\int_{0}^{T} \beta\left|u^{\prime}(s)\right|^{2} d s}{\int_{0}^{T} \beta|u(s)|^{2} d s}
$$

from which we deduce the first result. The remaining result is clear.

Corollary. -

$$
\lambda_{1}\left(\beta_{1}, \beta_{2}, 0, T\right)=\inf \left[\frac{\int_{0}^{T} \beta_{1}\left(v^{\prime}\right)^{2} d x}{\int_{0}^{T} \beta_{2} v^{2} d x} \mid v \in W\right] \geqslant \lambda_{1}(\beta, \beta, 0, T)
$$

where

$$
W=\{v \text { defined on }] 0, T\left[\mid \sqrt{\beta_{2}} v \text { and } \sqrt{\beta_{1}} v^{\prime} \in L^{2}(] 0, T[), v(T)=0\right\}
$$

Proof. - Let us give $v$ belonging to $W$. From (A.1), it is clear that $\sqrt{\beta} v$ and $\sqrt{\beta} v^{\prime}$ belong to $L^{2}$ (]0, $T[$ ), i.e., $W \subset V$. We have

$$
\begin{aligned}
& \frac{\int_{0}^{T} \beta_{1}\left(v^{\prime}\right)^{2} d x}{\int_{0}^{T} \beta_{2} v^{2} d x} \geqslant \frac{\int_{0}^{T} \beta\left(v^{\prime}\right)^{2} d x}{\int_{0}^{T} \beta v^{2} d x}, \quad \forall v \in W \subset V ; \\
& \frac{\int_{0}^{T} \beta_{1}\left(v^{\prime}\right)^{2} d x}{\int_{0}^{T} \beta_{2} v^{2} d x} \geqslant \lambda_{1}(\beta, \beta, 0, T), \quad \forall v \in W
\end{aligned}
$$

from which the result follows easily.

Proposition A.2. - For any $t, 0<t<T$ we have

$$
\lambda_{1}(\beta, \beta, t, T)>\lambda_{1}(\beta, \beta, 0, T) .
$$

Proof. - Let us remark that

$$
\lambda_{1}(\beta, \beta, t, T)=\inf \left[\frac{\int_{t}^{T} \beta\left(v^{\prime}\right)^{2} d x}{\int_{t}^{T} \beta v^{2} d x} \mid v \in H^{1}(] t, T[), v(T)=0\right]
$$


since $\beta$ is continuous and positive on $[t, T]$. Let us denote by $\mu_{1}(\beta, \beta, 0, T)$ the right hand side of (A.2). It is clear that we have

$$
\mu_{1}(\beta, \beta, t, T)>\mu_{1}(\beta, \beta, 0, T)=\inf \left[\frac{\int_{0}^{T} \beta\left(v^{\prime}\right)^{2} d x}{\int_{0}^{T} \beta v^{2} d x} \mid v \in H^{1}(] 0, T[), v(T)=0\right]
$$

since

$$
\forall v \in H^{1}(] 0, T[),\left.\quad v\right|_{] t, T[} \in H^{1}(] t, T[) .
$$

As

$$
\left\{v \in H^{1}(] 0, T[) \mid v(T)=0\right\} \subset V
$$

we can claim that

$$
\mu_{1}(\beta, \beta, 0, T)>\lambda_{1}(\beta, \beta, 0, T) .
$$

From (A.2) and (A.3) we deduce the result.

Remark. - An easy computation shows that

$$
\lambda_{1}(1,1,0, T)=\left(\frac{\pi}{2}\right)^{2} \cdot \frac{1}{T^{2}} .
$$

\section{Appendix B. A degenerate ordinary differential equation}

Let us consider the following equation:

$$
\left\{\begin{array}{l}
\left.-\left(\beta a^{\prime}\right)^{\prime}+\gamma \beta a=0 \quad \text { in }\right] 0, T[ \\
\left(\beta a^{\prime}\right)(0)=0, \quad a(T)=1,
\end{array}\right.
$$

where $\beta($.$) is a continuous, positive and nondecreasing function defined on [0, T]$ such that

$$
\beta(0)=0, \quad \beta(t) \geqslant c . t^{\alpha} \quad \forall t>0 ;
$$

$c$ and $\alpha$ are positive constants, $\gamma$ is a bounded function which has a constant sign in a neighbourhood of 0 . We suppose that

$$
\lambda_{1}(\beta, \beta, 0, T)>\gamma^{-}(t)+\eta \quad \text { a.e. } t \in[0, T],
$$

where $\eta$ is a positive constant, $\gamma^{-}(t)=-\inf (0, \gamma(t))$,

$$
\lambda_{1}(\beta, \beta, 0, T)=\inf \left[\frac{\int_{0}^{T} \beta\left(v^{\prime}\right)^{2} d x}{\int_{0}^{T} \beta v^{2} d x} \mid v \in V\right]
$$

with

$$
\left.\left.V=\left\{v \in L_{\mathrm{loc}}^{2}(] 0, T\right]\right) \mid \sqrt{\beta} v \text { and } \sqrt{\beta} v^{\prime} \in L^{2}(0, T), v(T)=0\right\},
$$




$$
\lambda_{1}(1,1,0, T)>\frac{T^{2}}{2}\|\gamma\|_{L^{\infty}(0, T)}^{2} .
$$

Proposition B.1. - Assume that $\gamma$ satisfies (B.2). Then Eq. (B.1) has a unique solution, denoted by $a_{\beta}($.$) , which is positive and belongs to V$. In addition $a_{\beta}($.$) belongs$ to $W^{1, \infty}(] 0, T[)$ if $\gamma$ is nonnegative in a neighbourhood of 0 . If $\gamma$ satisfies (B.3), the following estimate holds:

$$
\left\|a_{\beta}\right\|_{W^{1, \infty}(0, T)} \leqslant K
$$

where $K$ is a constant independent of $\beta$.

Proof. - If there is no ambiguity, we denote $a_{\beta}($.$) by a($.$) .$

First step. (Existence).

Let $b$ be a regular function on $[0, T]$ such that

$$
b^{\prime}(x)=0 \quad \forall x \in[0, \tau] \quad \text { for some } \tau>0, \quad b(T)=1,
$$

and let us set $k=\left[\left(\beta b^{\prime}\right)^{\prime}-\gamma \beta b\right] / \sqrt{\beta}$ and $a=u+b$. It is sufficient to prove the existence of a solution $u$ of

$$
\left\{\begin{array}{l}
\left.-\left(\beta u^{\prime}\right)^{\prime}+\gamma \beta u=k \quad \text { on }\right] 0, T[, \\
\left(\beta u^{\prime}\right)(0)=0, \quad u(T)=0 .
\end{array}\right.
$$

This equation has the following variational formulation:

$$
A(u, v)=\int_{0}^{T} \beta u^{\prime} v^{\prime} d x+\int_{0}^{T} \gamma \beta u v d x=\int_{0}^{T} k \sqrt{\beta} v d x \quad \forall v \in V .
$$

From Appendix A and since $\gamma$ verifies (2) Lax-Milgram theorem shows that there exists a unique solution $u$ of (B.3.1). And it is classical to see that $u$ is also solution of (B.2.1).

Second step. $(a(x) \geqslant 0 \forall x \in[0, T]$.

Let us remark that if $a$ belongs to $H^{1}(] 0, T[)$, the result is classical. It is obvious that $a$ belongs to $H^{2}$ (] $\varepsilon, T[) \forall \varepsilon>0$. Therefore $a$ and $a^{\prime}$ are continuous on ]0,T]. We have two cases to consider:

(i) first case: $\lim |a(t)|=+\infty$ as $t \rightarrow 0^{+}$;

(ii) second case: $\lim \left|a^{\prime}(t)\right|=+\infty$ as $t \rightarrow 0^{+}$.

(i) Let $\varepsilon>0$ be small enough to obtain that the sign function of $a$ is constant on $] 0, \varepsilon]$ and $|a(t)| \geqslant|a(\varepsilon)| \forall t \in] 0, \varepsilon]$. We argue by contradiction, i.e., we suppose that

$$
|\{x \mid a(x)<0\}|>0
$$

where $|F|$ stands for the Lebesgue measure of the measurable set $F$. We have

$$
\int_{0}^{T}\left(\lambda_{1}(\beta, \beta, 0, T)-\gamma^{-}(x)\right)\left(a^{-}\right)^{2} \beta(x) d x>0 .
$$


If we choose $\varepsilon$ small enough, we can assume that

$$
\int_{\varepsilon}^{T}\left[\lambda_{1}(\beta, \beta, 0, T)-\gamma^{-}\right] \beta\left(a^{-}\right)^{2} d x>\|\gamma\|_{\infty} \cdot \int_{0}^{\varepsilon} \beta a^{2} d x
$$

since $\int_{0}^{T} \beta a^{2} d x<+\infty$. Let us set

$$
\widetilde{a}(x)= \begin{cases}a(x) & \forall x \in[\varepsilon, T] \\ a(\varepsilon) & \forall x \in] 0, \varepsilon[\end{cases}
$$

it is clear that $\tilde{a} \in H^{1}(] 0, T\left[\right.$ ). After multiplying (B.1) by $\widetilde{a}^{-}$, and integrating by parts, we obtain

$$
\int_{0}^{T} \beta a^{\prime}\left(\widetilde{a}^{-}\right)^{\prime} d x+\int_{0}^{T} \beta \gamma a \widetilde{a}^{-} d x=0
$$

i.e.,

$$
\int_{\varepsilon}^{T} \beta a^{\prime}\left(a^{-}\right)^{\prime} d x-\int_{\varepsilon}^{T} \beta \gamma\left(a^{-}\right)^{2} d x-\int_{0}^{\varepsilon} \beta \gamma\left(a^{-}\right) \tilde{a}^{-} d x=0
$$

since $a$ and $\tilde{a}$ have the same sign in ]0, $\varepsilon$ ]. (B.6) can be written

$$
\int_{\varepsilon}^{T} \beta\left[\left(a^{-}\right)^{\prime}\right]^{2} d x+\int_{\varepsilon}^{T} \beta \gamma\left(a^{-}\right)^{2} d x+\int_{0}^{\varepsilon} \beta \gamma a^{-} \widetilde{a}^{-} d x=0 .
$$

But from Appendix A

$$
\begin{aligned}
\int_{\varepsilon}^{T} \beta\left[\left(a^{-}\right)^{\prime}\right]^{2} d x & \geqslant \lambda_{1}(\beta, \beta, \varepsilon, T) \cdot \int_{\varepsilon}^{T} \beta\left(a^{-}\right)^{2} d x \\
& \geqslant \lambda_{1}(\beta, \beta, 0, T) \cdot \int_{\varepsilon}^{T} \beta\left(a^{-}\right)^{2} d x .
\end{aligned}
$$

From (B.7) and (B.8) we can write

$$
\begin{gathered}
\int_{\varepsilon}^{T} \lambda_{1}(\beta, \beta, 0, T) \beta\left(a^{-}\right)^{2} d x+\int_{\varepsilon}^{T} \beta \gamma^{+}\left(a^{-}\right)^{2} d x \\
-\int_{\varepsilon}^{T} \beta \gamma^{-}\left(a^{-}\right)^{2} d x+\int_{0}^{\varepsilon} \beta \gamma a^{-} \widetilde{a}^{-} d x \leqslant 0,
\end{gathered}
$$

or again 


$$
\begin{aligned}
& \int_{\varepsilon}^{T}\left[\lambda_{1}(\beta, \beta, 0, T)-\gamma^{-}\right] \beta\left(\left(a^{-}\right)\right)^{2} d x-\|\gamma\|_{\infty} \int_{0}^{\varepsilon} \beta a^{2} d x \\
& \quad+\int_{\varepsilon}^{T} \beta \gamma^{+}\left(a^{-}\right)^{2} d x \leqslant 0,
\end{aligned}
$$

since $\left.\left.\left.\left|a^{-}(\varepsilon)\right|=\left|\widetilde{a}^{-}(s)\right| \leqslant|a(s)| \forall s \in\right] 0, \varepsilon\right],\left|a^{-}(s)\right| \leqslant|a(s)| \forall s \in\right] 0, T[$. contradicts (B.5). Therefore $a^{-}(x)=0$ a.e. $\left.\left.x \in\right] 0, T\right]$.

(ii) Since $\gamma$ has a constant sign in a neighbourhood of $0, a$ is monotone in neighbourhood of 0 , from (B.1). Thus the limit of $|a(t)|$, as $t$ goes to $0^{+}$, exists. And in this case we can suppose $\lim |a(t)|=\theta<+\infty$, as $t \rightarrow 0^{+}$.

As the function $a$ belongs to $\left.\left.C^{0}(] 0, T\right]\right)$, it is bounded on $[0, T]$. Hence we have

$$
\int_{0}^{T}|a(s)| d s<+\infty
$$

By (B.1) $\beta a^{\prime}$ is a continuous function on $[0, T]$ and

$$
\begin{aligned}
& \forall x>0 \quad a^{\prime}(x) \beta(x)=\int_{0}^{x} \gamma \beta a d s, \\
& \forall x>0 \quad\left|a^{\prime}(x)\right| \leqslant \frac{1}{\beta(x)} \int_{0}^{x}|\gamma| . \beta .|a| d s \leqslant\|\gamma\|_{\infty} \frac{1}{\beta(x)} \int_{0}^{x} \beta(x)|a| d s
\end{aligned}
$$

since $\beta($.$) is nondecreasing. Thus we have$

$$
\forall x>0, \quad\left|a^{\prime}(x)\right| \leqslant\|\gamma\|_{\infty} \cdot \int_{0}^{T}|a(s)| d s .
$$

And by (B.11) we obtain that $a$ belongs to $W^{1, \infty}(] 0, T[$ ). Now we can multiply (B.1) by $a^{-}$, and after integrating by parts we obtain:

$$
\begin{gathered}
-\int_{0}^{T} \beta\left[\left(a^{-}\right)^{\prime}\right]^{2} d x-\int_{0}^{T} \gamma \beta\left(a^{-}\right)^{2} d x=0, \\
\int_{0}^{T} \beta\left[\left(a^{-}\right)^{\prime}\right]^{2} d x-\int_{0}^{T} \gamma^{-} \beta\left(a^{-}\right)^{2} d x+\int_{0}^{T} \gamma^{+} \beta\left(a^{-}\right)^{2} d x=0 .
\end{gathered}
$$

From Proposition A.1, (B.12) becomes:

$$
\int_{0}^{T}\left[\lambda_{1}(\beta, \beta, 0, T)-\gamma^{-}\right] \beta\left(a^{-}\right)^{2} d x+\int_{0}^{T} \gamma^{+} \beta\left(a^{-}\right)^{2} d x \leqslant 0 .
$$


From (B.2), (B.13) entails that $a^{-}(x)=0$ a.e. $\left.\left.x \in\right] 0, T\right]$. This achieves the proof of the second step.

Third step. $(a>0$.

We first propose to prove that $\lim _{x \rightarrow 0^{+}} a(x)>0$. Then, in a second step, we will show that $a(x)>0 \forall x \in] 0, T]$.

(a) We argue by contradiction and we use Gronwall technique. Assume $a(0)=0$. From (B.1) we obtain

$$
\forall x>0 \quad a^{\prime}(x) \leqslant \frac{\|\gamma\|_{\infty}}{\beta(x)} . \int_{0}^{x} \beta(s) a(s) d s .
$$

Since $a(0)=0, a \in C^{0}([0, T])$ and (B.14) becomes

$$
\forall x>0 \quad a^{\prime}(x) \leqslant\|\gamma\|_{\infty} \int_{0}^{x} a(s) d s
$$

because $\beta($.) is nondecreasing. (B.15) can be written:

$$
\left\{\begin{array}{l}
U^{\prime \prime}(x) \leqslant\|\gamma\|_{\infty} \cdot U(x) \quad \forall x>0 \\
U(x) \geqslant 0, \quad U(0)=U^{\prime}(0)=0
\end{array}\right.
$$

where

$$
U(x)=\int_{0}^{x} a(s) d s \geqslant 0 .
$$

By integration (B.16) entails

$$
U^{\prime}(x) \leqslant\|\gamma\|_{\infty} \cdot \int_{0}^{x} U(s) d s .
$$

Thus we have

$$
\forall x>0 \quad \frac{U^{\prime}(x)}{U(x)} \leqslant\|\gamma\|_{\infty} \cdot \frac{\int_{0}^{x} U(s) d s}{U(x)} \leqslant\|\gamma\|_{\infty} \cdot x
$$

since $U($.$) is nondecreasing, i.e.,$

$$
\frac{U^{\prime}(x)}{U(x)} \leqslant\|\gamma\|_{\infty} \cdot x, \quad \forall x>0 .
$$

Let $t>0$ be arbitrary and $0<\varepsilon<t$. After integrating by parts on $[\varepsilon, t]$, (B.17) becomes

$$
0 \leqslant U(t) \leqslant U(\varepsilon) \exp \left[\frac{\|\gamma\|_{\infty}}{2}\left(t^{2}-\varepsilon^{2}\right)\right] \leqslant C \cdot U(\varepsilon) .
$$


Passing to the limit in (B.18), as $\varepsilon$ goes to zero, we obtain

$$
U(t)=0 \quad \forall t>0 \quad \text { i.e. } \quad a \equiv 0
$$

which contradicts (B.1). Thus $\lim a(x)>0$, as $x \rightarrow 0^{+}$.

(b) We now suppose that there exists $x_{0}>0$ such that $a\left(x_{0}\right)=0$. But $a(x) \geqslant 0$ for any $x$ in $] 0, T]$ and $a($.$) belongs to C^{1}(] 0, T[)$. This entails that we have

$$
a^{\prime}\left(x_{0}\right)=0 .
$$

Then, from (B.1), we can write

$$
\forall x>x_{0} \quad a^{\prime}(x) \leqslant\|\gamma\|_{\infty} \int_{x_{0}}^{x} a(s) d s .
$$

Then we proceed exactly as in (a) to conclude that

$$
a(x)=0 \quad \forall x, x_{0} \leqslant x \leqslant T .
$$

And this contradicts (B.1) since $a(T)=1$.

We point out that in previous steps we do not have necessarily the positivity of $\gamma$ in neighbourhood of 0 .

Fourth step. $\left(a \in W^{1, \infty}(] 0, T[)\right.$.)

Here we need to use that $\gamma$ is nonnegative in a neighbourhood of 0 , denoted by $\vartheta(0)=] 0, \tau]$. From (B.1), $\beta a^{\prime}($.$) is increasing in \vartheta(0)$. As $\left(\beta a^{\prime}\right)(0)=0$, we have

$$
\beta a^{\prime}(x) \geqslant 0 \quad \forall x \in \vartheta(0),
$$

i.e.,

$$
a^{\prime}(x) \geqslant 0 \quad \forall x \in \vartheta(0) .
$$

Hence $a$ is increasing in $] 0, \tau]$. Therefore $\lim _{x \rightarrow 0^{+}} a(x)$ exists and we have

$$
0 \leqslant \lim _{x \rightarrow 0^{+}} a(x) \leqslant a(\tau)<+\infty .
$$

Since $\left.\left.a \in C^{0}(] 0, T\right]\right)$ we can set

$$
a(0)=\lim _{x \rightarrow 0^{+}} a(x),
$$

and $a(0)>0$, by the third step. Consequently $a($.$) belongs to C^{0}([0, T])$ and from (B.1) we obtain:

$$
\forall x>0, \quad\left|a^{\prime}(x)\right| \leqslant \frac{1}{\beta(x)} \int_{0}^{x}|\gamma| \beta(s) a(s) \leqslant\|\gamma\|_{\infty} \cdot \int_{0}^{x} a(s) d s .
$$


Thus

$$
\forall x>0, \quad\left|a^{\prime}(x)\right| \leqslant\|\gamma\|_{\infty} \cdot\|a\|_{\infty} .
$$

This proves that $a \in W^{1, \infty}(] 0, T[)$.

Fifth step. $\left(\left\|a_{\beta}\right\|_{W^{1, \infty}(0, T)} \leqslant K\right.$.)

Now let us prove the estimate

$$
\left\|a_{\beta}\right\|_{W^{1, \infty}(0, T)} \leqslant K=\text { constant independent of } \beta .
$$

We have:

$$
\left|a_{\beta}^{\prime}(x)\right| \leqslant\|\gamma\|_{\infty} \cdot \int_{0}^{x} a_{\beta}(s) d s \leqslant\|\gamma\|_{\infty} \cdot \sqrt{x}\left(\int_{0}^{T}\left|a_{\beta}\right|^{2} d s\right)^{1 / 2},
$$

by Cauchy-Schwarz. We have

$$
\int_{0}^{T}\left|a_{\beta}^{\prime}\right|^{2} d s \leqslant\|\gamma\|_{\infty}^{2} \frac{T^{2}}{2} \int_{0}^{T}\left|a_{\beta}\right|^{2} d s .
$$

By Poincaré inequality (cf. Appendix A) we have

$$
\begin{aligned}
& \lambda_{1}(1,1,0, T) \int_{0}^{T}\left|a_{\beta}-1\right|^{2} d s \leqslant\|\gamma\|_{\infty}^{2} \cdot \frac{T^{2}}{2} \cdot \int_{0}^{T}\left|a_{\beta}\right|^{2} d s \\
& \left(\lambda_{1}(1,1,0, T)-\|\gamma\|_{\infty}^{2} \cdot \frac{T^{2}}{2}\right) \cdot \int_{0}^{T}\left|a_{\beta}\right|^{2} d s \\
& \quad \leqslant 2 \lambda_{1}(1,1,0, T) \int_{0}^{T}\left|a_{\beta}\right| d s \leqslant 2 \lambda_{1}(1,1,0, T) \sqrt{T}\left(\int_{0}^{T}\left|a_{\beta}\right|^{2} d s\right)^{1 / 2},
\end{aligned}
$$

or again

$$
\left\|a_{\beta}\right\|_{L^{2}} \leqslant \frac{2 \lambda_{1}(1,1,0, T) \sqrt{T}}{\lambda_{1}(1,1,0, T)-\|\gamma\|_{\infty}^{2} \cdot T^{2} / 2} .
$$

From (B.19) and (B.20) we deduce easily that

$$
\left\|a_{\beta}\right\|_{\infty} \leqslant C, \quad\left\|a_{\beta}^{\prime}\right\|_{\infty} \leqslant C
$$

where $C$ denotes some constant independent of $\beta$. Now the proof of Proposition 1 is complete.

\section{B.1. An other regularity result}

Let us consider the problem studied previously. Let us assume that $\gamma$ verifies inequality (B.2) and take $\beta(t)=c . t^{\alpha}, \alpha>0$. We suppose that there exists $r_{0}>0$ such 
that

$$
\left\{\begin{array}{l}
|\gamma(r)| \leqslant d . r^{\theta} \quad \forall r \in\left[0, r_{0}\right] \\
\theta>\frac{\alpha-1}{2} .
\end{array}\right.
$$

PROPOSITION B.2. - If $\gamma$ satisfies (B.21), then the solution a of following equation

$$
\left\{\begin{array}{l}
-\frac{1}{r^{\alpha}}\left(r^{\alpha} a^{\prime}\right)^{\prime}+\gamma a=0 \quad \text { in }(] 0, T[), \\
\left(r^{\alpha} a^{\prime}\right)(0)=0, \quad a(T)=1
\end{array}\right.
$$

belongs to $W^{1, \infty}(] 0, T[)$. In addition $a^{\prime}(0)=0$.

Proof. - From Proposition 1 there exists a unique function $a$ such that $r^{\alpha / 2} a(r)$ and $r^{\alpha / 2} a^{\prime}(r)$ belong to $L^{2}(] 0, T[)$. Thus for any $r, 0<r<r_{0}$, we have

$$
\begin{gathered}
r^{\alpha} a^{\prime}(r)=\int_{0}^{r} s^{\alpha} \gamma(s) a(s) d s, \\
\forall r>0, \quad\left|a^{\prime}(r)\right| \leqslant \frac{1}{r^{\alpha}} \int_{0}^{r} s^{\alpha}|\gamma(s)||a(s)| d s, \\
\left|a^{\prime}(r)\right| \leqslant d . \int_{0}^{r} s^{\theta}|a(s)| d s \quad \forall r, 0<r<r_{0} .
\end{gathered}
$$

Using Cauchy-Schwarz, we obtain

$$
\forall r, 0<r<r_{0}, \quad\left|a^{\prime}(r)\right| \leqslant d \cdot \frac{r^{2 \theta-\alpha+1}}{2 \theta-\alpha+1} \cdot\left(\int_{0}^{T} s^{\alpha}|a(s)|^{2} d s\right)^{1 / 2} .
$$

As $a^{\prime}$ is continuous on ]0, $T$ [, we have

$$
\lim _{r \rightarrow O^{+}}\left|a^{\prime}(r)\right|=0
$$

i.e., we can set, by definition, $a^{\prime}(0)=0$, since $a \in C^{1}(] 0, T$ [). Inequality (B.22) entails that $a^{\prime}$ belongs to $L^{\infty}(] 0, T[)$; and since

$$
a(r)=\int_{r}^{T} a^{\prime}(s) d s+1,
$$

we can claim that $a$ belongs to $W^{1, \infty}(] 0, T[)$.

Remark. - Assume that $\gamma$ verifies $\left(\mathrm{H}^{\prime} 2\right)$. Let us give $\beta\left(\right.$.) such that $\beta(t) \sim t^{\alpha}$ in a neighbourhood of 0 . We can prove that the solution $a_{\beta}$ of

$$
\left\{\begin{array}{l}
\left.-\frac{1}{\beta}\left(\beta a_{\beta}^{\prime}\right)^{\prime}+\gamma a_{\beta}=0 \quad \text { in }\right] 0, T[ \\
\left(\beta a_{\beta}^{\prime}\right)(0)=0, \quad a(T)=0,
\end{array}\right.
$$


belongs to $W^{1, \infty}(] 0, T[)$ and verifies $a_{\beta}^{\prime}(0)=0$. In addition, proceeding as in the proof of Proposition 1, we can state that $\left\|a_{\beta}\right\|_{W^{1, \infty}} \leqslant C, C$ is constant independent of $\beta$.

\section{Appendix C. Extension result}

Let $\Omega$ be a bounded open set of $\mathbb{R}^{N}$, and $\Theta$ be an open regular subset of $\Omega$. Consider the operator

$$
A=-\sum_{i j} \frac{\partial}{\partial x_{i}}\left(\alpha_{i j} \frac{\partial}{\partial x_{i}}\right)+\sum_{i} \beta_{i} \frac{\partial}{\partial x_{i}}+c .
$$

We suppose that $A$ is uniformly elliptic:

$$
\left\{\begin{array}{l}
\sum_{i j} \alpha_{i j}(x) \xi_{i} \xi_{j} \geqslant \alpha|\xi|^{2} \quad \text { a.e. } x \in \Omega, \forall \xi \in \mathbb{R}^{N}, \\
\alpha \text { is a constant }>0, \quad \alpha_{i j}=\alpha_{j i} \forall i, j .
\end{array}\right.
$$

Let $v$ be the solution of the following equation

$$
\left\{\begin{array}{l}
A v=f \quad \text { in } \Theta \\
\left.v\right|_{\partial \Theta}=0
\end{array}\right.
$$

where $f$ belongs to $L^{2}(\Theta)$.

EXTENSION LEMMA. - Suppose that the solution $v$ of (C.1) satisfies

$$
\sum_{i, j} \alpha_{i j} \frac{\partial v}{\partial x j} \cos \left(n, x_{i}\right) \mid \partial \Theta \leqslant 0 \quad \text { in } H^{-1 / 2}(\partial \Theta),
$$

where $n$ stands for the outer normal to $\partial \Theta$. Then we have:

$$
A \widetilde{v} \leqslant \widetilde{f} \text { in the sense of } H^{-1}(\Omega),
$$

where, for any function s defined on $\Theta, \widetilde{s}$ stands for the extension of s by zero, to $\Omega$.

Proof. - It is based on Green formula. For any nonnegative function $\varphi$ belonging to $\mathcal{D}(\Omega)$, we have, integrating by parts:

$$
\begin{aligned}
\left\langle-\sum_{i, j} \frac{\partial}{\partial x_{i}} \alpha_{i j} \frac{\partial \widetilde{v}}{\partial x_{j}}, \varphi\right\rangle_{H^{-1}(\Omega) \times H_{0}^{1}(\Omega)}= & \left\langle-\sum_{i, j} \frac{\partial}{\partial x_{j}} \alpha_{i j} \frac{\partial \varphi}{\partial x_{i}}, \widetilde{v}\right\rangle_{\mathcal{D}^{\prime}(\Omega) \times \mathcal{D}(\Omega)} \\
= & \int_{\Theta}-\sum_{i j}\left(\frac{\partial}{\partial x_{i}} \alpha_{i j} \frac{\partial v}{\partial x_{j}}\right) \cdot \varphi d x \\
& +\int_{\partial \Theta} \sum_{i j} \alpha_{i j} \frac{\partial v}{\partial x_{j}} \cos \left(n, x_{i}\right) \varphi d \sigma \\
\leqslant & \int_{\Theta}-\sum_{i j}\left(\frac{\partial}{\partial x_{i}} \alpha_{i j} \frac{\partial v}{\partial x_{j}}\right) \varphi d x,
\end{aligned}
$$


thanks hypothesis (C.2) and since $\tilde{v} \in H_{0}^{1}(\Omega)$, with

$$
\nabla \tilde{v}(x)= \begin{cases}\nabla v(x) & \text { a.e. in } \Theta \\ 0 & \text { in } \Omega \backslash \bar{\Theta} .\end{cases}
$$

From (C.4) it follows

$$
\begin{aligned}
\int_{\Omega} \sum_{i} \beta_{i} \frac{\partial \tilde{v}}{\partial x_{i}} \varphi d x & =\int_{\Theta} \sum_{i} \beta_{i} \frac{\partial v}{\partial x_{i}} \varphi d x, \\
\int_{\Omega} c \tilde{v} \varphi d x & =\int_{\Theta} c v \varphi d x .
\end{aligned}
$$

Finally (C.3), (C.5) and (C.6) give

$$
\langle A \widetilde{v}, \varphi\rangle_{H^{-1}(\Omega) \times H_{0}^{1}(\Omega)} \leqslant \int_{\Theta} A v \varphi d x=\int_{\Theta} f \varphi d x=\int_{\Omega} \tilde{f} \varphi d x,
$$

for any nonnegative $\varphi$ belonging to $H_{0}^{1}(\Omega)$. That is $A \widetilde{v} \leqslant \widetilde{f}$ in sense of $H^{-1}(\Omega)$.

\section{REFERENCES}

[1] H. Brezis, Analyse fonctionnelle. Théorie et applications, Masson, 1983.

[2] K.M. Chong, N.M. Rice, Equimeasurable Rearrangements of Functions, in: Queen's Papers in Pure and Applied Mathematics, Vol. 28, Queen's University, Ontario, 1971.

[3] J.I. Diaz, B. Kawhol, On convexity and starshapdness of level sets for some nonlinear elliptic and parabolic problems on convex rings, J. Math. Anal. Appl. 177 (1993) 263286.

[4] L.C. Evans, Partial Differential Equations, Graduate Studies in Mathematics, Vol. 19.

[5] D. Gilbarg, N.S. Trudinger, Elliptic Partial Differential Equations of Second Order, Springer Verlag, 1983.

[6] B. Kawohl, Rearrangements and Convexity of Level Sets in PDE, in: Lecture Notes in Math., Vol. 1150, Springer, 1985.

[7] J.L. Lewis, Capacitary functions in convex rings, Arch. Rational Mech. Anal. 66 (1977) 201-224.

[8] J. Mossino, Inégalités isopérimétriques et applications en physique. Travaux en cours, Hermann, Paris, 1984.

[9] M. Protter, H. Weinberger, Maximum Principles in Differential Equations, Prentice-Hall, 1967.

[10] R. Sperb, Maximum Principles and Their Applications, Academic Press, 1981.

[11] G. Stampacchia, Le problème de Dirichlet pour les équations elliptiques du second ordre à coefficients discontinus, Ann. Inst. Fourier 15 (1965) 189-258.

[12] R. Tahraoui, Contrôle optimal dans les équations elliptiques, SIAM J. Control Optim. 3 (1992) 465-521.

[13] R. Tahraoui, Sur le principe du maximum des opérateurs elliptiques, C. R. Acad. Sci. Paris, Série I 320 (1995) 1453-1458. 
[14] R. Tahraoui, Générateurs infinitésimaux et propriétés géométriques pour certaines équations complètement non linéaires, Revista Matemática Iberoamericana 11 (3) (1995).

[15] R. Tahraoui, Principe de comparaison pour opérateurs elliptiques, C. R. Acad. Sci. Paris, Série I 322 (1996) 1053-1056.

[16] R. Tahraoui, Star-shapedeness of solutions of some semi-linear problems, Work in preparation.

[17] L. Tartar, Estimations fines des coefficients homogénéisés, in: Ennio de Giorgi Colloquium, Vol. 125, Pitman, pp. 168-187.

[18] D.L. Cohn, Measure Theory, Birkhäuser, Boston, 1980.

[19] F. Flores-Bazán, A. Cellina, Radially symmetric solutions of a class of problems of the calculus of variations without convexity assumptions, Ann. I. H. Poincaré AN 9 (1992) $465-478$. 